\title{
OPTIMAL BOUNDS ON THE EFFECTIVE BEHAVIOR OF A MIXTURE OF TWO WELL-ORDERED ELASTIC MATERIALS
}

\author{
BY \\ GREGOIRE ALLAIRE' (Commissariat a l'Energie Atomique, Gif sur Yvette, France) \\ AND \\ ROBERT V. KOHN ${ }^{2}$ (Courant Institute of Mathematical Sciences, New York, New York)
}

\begin{abstract}
We consider linearly elastic composite materials made by mixing two possibly anisotropic components. Our main hypothesis is that the Hooke's laws of the two components be well-ordered. For given volume fractions and average strain, we present optimal upper and lower bounds on the elastic energy quadratic form. We also discuss bounds on sums of energies and bounds involving complementary energy rather than elastic energy. Our arguments are based primarily on the HashinShtrikman variational principle; however, we also discuss how the same results arise from the "translation method", making use of the analysis of Milton. Our bounds are equivalent to those established by Avelleneda and closely related to the "trace bounds" established by Milton and Kohn. The optimal energy bounds, however, are presented here as the extreme values of certain convex optimization problems. The optimal microgeometries are determined by the associated first-order optimality conditions. A similar treatment for mixtures of two incompressible, isotropic elastic materials has previously been given by Kohn and Lipton.
\end{abstract}

1. Introduction. The macroscopic properties of a linearly elastic composite are described by its tensor of effective moduli (Hooke's law) $\sigma^{*}$. This fourth-order tensor depends on the microgeometry of the mixture and on the elastic properties of the components.

Suppose that $\sigma^{*}$ arises by mixing two materials $\sigma_{1}$ and $\sigma_{2}$ with volume fractions $\theta_{1}$ and $\theta_{2}$, respectively. It is well known that $\sigma^{*}$ must satisfy Paul's bounds, also called the Voigt-Reuss bounds:

$$
\left\langle\left(\theta_{1} \sigma_{1}^{-1}+\theta_{2} \sigma_{2}^{-1}\right)^{-1} \xi, \xi\right\rangle \leq\left\langle\sigma^{*} \xi, \xi\right\rangle \leq\left\langle\left(\theta_{1} \sigma_{1}+\theta_{2} \sigma_{2}\right) \xi, \xi\right\rangle
$$

Received June 17, 1991.

1991 Mathematics Subject Classification. Primary 73B27, 73K40.

${ }^{1}$ This work was begun while G. A. was a Visiting Member at the Courant Institute, supported by DARPA contract F49620-87-C-0065.

${ }^{2}$ The work of R. V. K. was partially supported by DARPA contract F-49620-87-C-0065, NSF grant DMS-8701895, ONR grant N00014-88-K-0279, ARO contract DAAL03-89-K-0039, and AFOSR grant 90-0090.

(C)1993 Brown University 
for any symmetric, second-order tensor $\xi$. These bounds, however, are not optimal; for most choices of $\xi$ it is impossible to saturate either inequality. Thus it is natural to seek bounds that improve on (1.1).

Recent work has led to results that not only improve upon (1.1) but do so optimally. By optimal bounds on $\left\langle\sigma^{*} \xi, \xi\right\rangle$, we mean a pair of functions $f_{ \pm}=$ $f_{ \pm}\left(\sigma_{1}, \sigma_{2}, \theta_{1}, \theta_{2}, \xi\right)$ such that

$$
f_{-} \leq\left\langle\sigma^{*} \xi, \xi\right\rangle \leq f_{+}
$$

and such that each inequality can be saturated (for any $\xi$ ) by a microstructure (which depends on $\xi$ ). Optimal bounds of this type have been established for mixtures of two incompressible isotropic elastic materials in [22], for mixtures of two wellordered isotropic materials in $[4,5]$, and for the analogous problem of plate theory in [13]. Avellaneda's work [4, 5] also discusses the optimal upper and lower bounds for a sum of elastic energies, i.e., bounds of the type

$$
f_{-} \leq\left\langle\sigma^{*} \xi_{1}, \xi_{1}\right\rangle+\cdots+\left\langle\sigma^{*} \xi_{N}, \xi_{N}\right\rangle \leq f_{+},
$$

where $\xi_{1}, \ldots, \xi_{N}$ are specified second-order tensors. It is equally natural to consider bounds on complementary energy, e.g.,

$$
f_{-} \leq\left\langle\left(\sigma^{*}\right)^{-1} \xi_{1}, \xi_{1}\right\rangle+\cdots+\left\langle\left(\sigma^{*}\right)^{-1} \xi_{N}, \xi_{N}\right\rangle \leq f_{+},
$$

and this, too, is addressed in $[4,5]$.

The literature just cited has some gaps and deficiencies, however, which this paper is designed to rectify. First, most attention has been devoted to the case when each component material is isotropic. It is well known to experts that similar results hold for mixtures of two well-ordered anisotropic materials, but this fact is difficult to glean from the literature. The presentation given here, by contrast, places no symmetry hypothesis on $\sigma_{1}$ or $\sigma_{2}$. Second, the analysis of $[4,5]$ gives $f_{+}$and $f_{-}$as the extreme values of certain nonconvex optimizations in a rather high-dimensional space. That approach, though elegant in concept, makes the task of actually evaluating the bounds rather difficult. Our analysis gives $f_{+}$and $f_{-}$as the extreme values of certain convex optimizations. For the bounds on a single energy (1.2), this optimization is over the relatively low-dimensional space of second-order tensors. Third, the optimality of these bounds depends on a link between the Hashin-Shtrikman variational principle and the formula for the effective moduli of a sequentially laminated composite. In most prior treatments this link is obtained by "inspection". Here, by contrast, the link is explained by the observation that the Hashin-Shtrikman principle is exact for a laminated composite. (A similar explanation can also be found in [28].)

Our method, which is based on the Hashin-Shtrikman variational principle, requires that the Hooke's laws of the component materials be well-ordered. This means that

$$
\left\langle\sigma_{1} \eta, \eta\right\rangle \leq\left\langle\sigma_{2} \eta, \eta\right\rangle
$$

for every second-order tensor $\eta$. When $\sigma_{1}$ and $\sigma_{2}$ are isotropic, (1.5) requires that the smaller bulk and shear moduli belong to the same material $\left(\sigma_{1}\right)$. We do not assume that $\sigma_{1}$ and $\sigma_{2}$ are isotropic. If they are anisotropic, however, their 
orientations are understood to be fixed; thus we are not discussing polycrystalline composites.

It should be emphasized that the symmetry of the effective Hooke's law $\sigma^{*}$ is not restricted in (1.2)-(1.4). Tighter bounds obtained by restricting the symmetry of $\sigma^{*}$ will be found in $[24,25]$.

Besides offering an alternative viewpoint on optimal energy bounds, we also discuss the translation method (Sec. 4), and the pairwise equivalence of upper and lower bounds for energies and complementary energies (Sec. 8). It was Milton who first found a link between the Hashin-Shtrikman variational principle and the newer translation method [28]. Section 4 specializes his argument to the case of the energy bound (1.2), which is much more transparent than the general case. The pairwise equivalence of the four different Hashin-Shtrikman bounds was observed in [29] for mixtures of well-ordered materials. That result is generalized in Sec. 8, using a new argument that requires neither well-ordering nor any specific representation for the optimal bounds.

There are several specific reasons to seek optimal bounds of the type (1.2)-(1.4). The optimal lower bound $\left\langle\sigma^{*} \xi, \xi\right\rangle \geq f_{-}$has recently been used by Francfort and Marigo in modelling the accumulation of damage [10]. The optimal lower bound on complementary energy $\left\langle\left(\sigma^{*}\right)^{-1} \xi, \xi\right\rangle \geq f_{-}$is central to our recent work on structural optimization [3]. After extension to permit the component materials to have different stress free strains, the optimal lower bound $\left\langle\sigma^{*} \xi, \xi\right\rangle \geq f_{-}$is also relevant to the calculation of phase diagrams for coherent mixtures of crystalline solids [20, 21]. From a broader perspective, optimal bounds arise in the " $G$-closure problem", which seeks a characterization of all composites $\sigma^{*}$ achievable as mixtures of $\sigma_{1}$ and $\sigma_{2}$ in specified volume fractions $\theta_{1}, \theta_{2}$. The optimal energy bounds (1.2)-(1.4) offer a partial solution, by specifying the extreme values of certain linear functions of $\sigma^{*}$.

The optimal energy bounds (1.2)-(1.4) can also be viewed as extensions of the well-known Hashin-Shtrikman bounds on the effective bulk and shear moduli of an isotropic composite. In their now classic work [15], Hashin and Shtrikman considered isotropic mixtures $\sigma^{*}$ made from two well-ordered isotropic components. They established upper and lower bounds on the effective bulk modulus $\kappa^{*}$ and shear modulus $\mu^{*}$. Both bounds are now known to be optimal [11, 27, 32]. Several authors have extended these results to bounds on the "generalized bulk modulus" and "generalized shear modulus" of a possibly anisotropic composite [11, 17, 29, 43]. Two possible definitions of the generalized bulk modulus are

$$
\frac{1}{N^{2}}\left\langle\sigma^{*} I, I\right\rangle \text { or }\left\langle\left(\sigma^{*}\right)^{-1} I, I\right\rangle^{-1},
$$

where $N$ is the spatial dimension and $I$ is the second-order identity tensor. Thus the optimal upper and lower bounds on a single energy $\left\langle\sigma^{*} \xi, \xi\right\rangle$ or complementary energy $\left\langle\left(\sigma^{*}\right)^{-1} \xi, \xi\right\rangle$ represent extensions of the generalized bulk modulus bounds. Two possible definitions of the generalized shear modulus are

$$
\frac{1}{2 d} \sum_{i=1}^{d}\left\langle\sigma^{*} \xi_{i}, \xi_{i}\right\rangle \text { or } \frac{d}{2}\left(\sum_{i=1}^{d}\left\langle\left(\sigma^{*}\right)^{-1} \xi_{i}, \xi_{i}\right\rangle\right)^{-1},
$$


where $\left\{\xi_{i}\right\}_{i=1}^{d}$ is an orthonormal basis for the space of trace-free second-order tensors. Thus, bounds of the type (1.3) or (1.4) can be viewed as extensions of the generalized shear modulus bounds.

The bounds presented here are in principle computable, but they are not explicit; we do not attempt to solve the relevant convex optimizations analytically. Explicit bounds of the type (1.2) are available, however, for isotropic $\sigma_{1}$ and $\sigma_{2}$ in the following cases: (i) both materials are incompressible [22]; (ii) the spatial dimension is $N=2$ [1, 13]; and (iii) the spatial dimension is $N=3$ and $\sigma_{1}=0[3,13]$.

It is natural to ask whether similar results might hold without the hypothesis of well-ordering (1.5). In two space dimensions, if $\sigma_{1}$ and $\sigma_{2}$ are isotropic, then the optimal energy bound (1.2) is known explicitly even in the non-well-ordered case [1, 13]. Our forthcoming paper [2] is concerned with the optimal lower bound $\left\langle\sigma_{*} \xi, \xi\right\rangle \geq$ $f_{-}$, for mixtures of two non-well-ordered isotropic materials in three (or more) space dimensions.

Other extensions of these results are to mixtures of materials with different stressfree strains [21] and to mixtures of a linearly elastic material with a physically nonlinear one.

The remainder of this introduction is devoted to establishing notation and reviewing basic facts about composite materials. We identify an elastic material with its Hooke's law $\sigma$, a positive definite symmetric tensor mapping symmetric (strain) tensors to symmetric (stress) tensors. There are several different ways to formalize the notion of a composite material made by mixing $\sigma_{1}$ and $\sigma_{2}$ in volume fractions $\theta_{1}$ and $\theta_{2}\left(\theta_{1} \geq 0, \theta_{2} \geq 0, \theta_{1}+\theta_{2}=1\right)$. One can use the spatially periodic theory (see, e.g., [6,35]), the theory of random composites (see, e.g., [14, 33]), or the theory of $G$-convergence (also known as $H$-convergence, see, e.g., $[11,30,39,44]$ ). The spatially periodic viewpoint is the easiest to work with. Moreover, it is sufficient for the purpose of proving bounds. (For rigorous proofs of this assertion, see [14] in the random setting and [8] in the context of $G$-convergence.) We shall therefore prove our bounds in the spatially periodic setting only.

A spatially periodic composite is described as follows. Let $Q=[0,1]^{N}$ be the unit cube in physical space. The microstructure is determined by $Q$-periodic functions $\chi_{1}(y)$ and $\chi_{2}(y)$, with

$$
\chi_{1}(y)=0 \text { or } 1 \text { almost everywhere and } \chi_{2}(y)=1-\chi_{1}(y) \text {. }
$$

The locally varying Hooke's law is

$$
\sigma_{\varepsilon}(y)=\chi_{1}\left(\frac{x}{\varepsilon}\right) \sigma_{1}+\chi_{2}\left(\frac{x}{\varepsilon}\right) \sigma_{2},
$$

where $\sigma_{1}$ and $\sigma_{2}$ are the Hooke's laws of the two components and $\varepsilon$ is the length scale of the microstructure. The volume fraction of material $i$ is evidently

$$
\theta_{i}=\int_{Q} \chi_{i}(y) d y, \quad i=1,2 .
$$

The effective Hooke's law $\sigma^{*}$ of the composite is characterized by

$$
\left\langle\sigma^{*} \xi, \xi\right\rangle=\inf _{\phi} \int_{Q}\langle\sigma(y)[\xi+e(\phi)], \xi+e(\phi)\rangle d y,
$$


where $\phi$ ranges over $Q$-periodic "elastic displacements", $e(\phi)$ represents $\frac{1}{2}\left(\nabla \phi+{ }^{t} \nabla \phi\right)$, and $\sigma(y)$ is the "microscopic Hooke's law"

$$
\sigma(y)=\chi_{1}(y) \sigma_{1}+\chi_{2}(y) \sigma_{2} .
$$

Its physical meaning is as follows (see, e.g., $[6,35]$ ). Consider a boundary-value problem involving a region $\Omega$ occupied by the composite, for example,

$$
\begin{gathered}
e\left(u_{\varepsilon}\right)=\frac{1}{2}\left(\nabla u_{\varepsilon}+{ }^{t} \nabla u_{\varepsilon}\right), \\
\nabla \cdot\left(\sigma_{\varepsilon} e\left(u_{\varepsilon}\right)=f \text { in } \Omega,\right. \\
u_{\varepsilon}=0 \text { at } \partial \Omega .
\end{gathered}
$$

As the length-scale $\varepsilon$ tends to zero, the solution $u_{\varepsilon}$ converges to that of the effective equation

$$
\begin{gathered}
e(u)=\frac{1}{2}\left(\nabla u+{ }^{t} \nabla u\right), \\
\nabla \cdot\left(\sigma^{*} e(u)\right)=f \text { in } \Omega, \\
u=0 \text { at } \partial \Omega .
\end{gathered}
$$

Thus $\sigma^{*}$ describes effective or macroscopic behavior as the length scale of the microstructure tends to zero.

We note some ambiguity in our use of the word "microstructure". Sometimes, as above after (1.7), this term refers to a spatially periodic mixture of $\sigma_{1}$ and $\sigma_{2}$. Elsewhere, as above following (1.2), it refers to a limit of spatially periodic compositesor equivalently, by virtue of [8], to a composite in the sense of $G$-convergence.

We are concerned with optimal bounds on $\sigma^{*}$, when $\sigma_{1}, \sigma_{2}, \theta_{1}$ and $\theta_{2}$ are held fixed, as the microstructure varies.

2. The Hashin-Shtrikman lower bound. We focus initially on lower bounds for the elastic energy quadratic form $\left\langle\sigma^{*} \xi, \xi\right\rangle$. Lower bounds on sums of energies are discussed in Sec. 4; upper bounds and complementary energy bounds are the focus of Sec. 5 .

We assume that $\sigma_{1}$ and $\sigma_{2}$ are strictly well-ordered, i.e., $\sigma_{1}<\sigma_{2}$ in the sense that

$$
\left\langle\sigma_{1} \eta, \eta\right\rangle\left\langle\left\langle\sigma_{2} \eta, \eta\right\rangle \quad \text { for all } \eta \neq 0 .\right.
$$

The hypothesis of strict well-ordering rather than the weaker $\sigma_{1} \leq \sigma_{2}$ is entirely a matter of technical convenience. All our results actually extend to the nonstrictly well-ordered case. One approach is to repeat the arguments presented here with appropriate care (see Remarks 2.2 and 3.6.) Another approach involves perturbing to the strictly well-ordered case and then passing to the limit in the bound (see Proposition 8.1).

We work throughout in an $N$-dimensional physical space, to encompass both two- and three-dimensional elasticity simultaneously. Let $S$ be the $[N(N+1) / 2]$ dimensional space of $N \times N$ symmetric tensors, with the usual inner product $\langle\xi, \eta\rangle=$ $\operatorname{tr}(\xi \eta)$. For any subspace $V$ of $S$, we write $\pi_{V} \xi$ for the orthogonal projection of $\xi$ onto $V$. If $k \in \mathbf{R}^{N}, k \neq 0$, we set

$$
V(k)=\left\{k \otimes \nu+\nu \otimes k: \nu \in \mathbf{R}^{N}\right\}
$$


it is an $N$-dimensional subspace of $S$. These subspaces play a special role, because $V(k)$ is the space of Fourier transforms of strains at frequency $k$. Notice that $V(k)$ really depends only on $k /|k|$.

Our version of the Hashin-Shtrikman lower bound is presented as Proposition 2.1. Its basis is the celebrated Hashin-Shtrikman variational principle. First introduced by Hashin and Shtrikman in [15], this method for bounding $\sigma^{*}$ has since been elaborated upon by many authors, including Hill [16], Walpole [40], and Willis [41]. Those authors controlled the "nonlocal term" through hypotheses on the symmetry of $\sigma^{*}$, coupled with properties of the elastic Green's function. We handle the nonlocal term differently, using Fourier analysis. This approach, developed in [29], has the advantage of requiring no hypotheses on the symmetry of $\sigma^{*}$. A viewpoint closely related to ours has been used by $\mathrm{Wu}$ and $\mathrm{McCullough}$ [42].

Proposition 2.1. Assume that $\sigma_{1}<\sigma_{2}$. Let $\sigma^{*}$ be the effective Hooke's law of a composite made from $\sigma_{1}$ and $\sigma_{2}$, in volume fractions $\theta_{1}$ and $\theta_{2}$, respectively. Then we have the following lower bound, for any $\xi \in S$ :

$$
\left\langle\sigma^{*} \xi, \xi\right\rangle \geq\left\langle\sigma_{1} \xi, \xi>+\theta_{2} \sup _{\eta}\left[2\langle\xi, \eta\rangle-\left\langle\left(\sigma_{2}-\sigma_{1}\right)^{-1} \eta, \eta\right\rangle-\theta_{1} g(\eta)\right],\right.
$$

in which the function $g(\eta)$ is defined by

$$
g(\eta)=\sup _{|k|=1}\left|\pi_{\sigma_{1}^{1 / 2} V(k)} \sigma_{1}^{-\frac{1}{2}} \eta\right|^{2} .
$$

Proof. We start from the definition of $\sigma^{*},(1.9)$. Adding and subtracting the "reference energy" $\left\langle\sigma_{1}(\xi+e(\phi)), \xi+e(\phi)\right\rangle$ gives

$$
\left\langle\sigma^{*} \xi, \xi\right\rangle=\inf _{\phi}\left[\int_{Q}\left\langle\left(\sigma(y)-\sigma_{1}\right)(\xi+e(\phi)), \xi+e(\phi)\right\rangle+\int_{Q}\left\langle\sigma_{1}(\xi+e(\phi)), \xi+e(\phi)\right\rangle\right] .
$$

Let us rewrite the first term on the right-hand side, using the positivity of $\sigma_{2}-\sigma_{1}$ and "convex duality":

$$
\begin{aligned}
\int_{Q}\left\langle\left(\sigma(y)-\sigma_{1}\right)(\xi+e(\phi)),\right. & \xi+e(\phi)\rangle=\int_{Q} \chi_{2}\left\langle\left(\sigma_{2}-\sigma_{1}\right)(\xi+e(\phi)), \xi+e(\phi)\right\rangle \\
& =\sup _{\tilde{\eta}(y)} \int_{Q} 2 \chi_{2}\langle\tilde{\eta}, \xi+e(\phi)\rangle-\int_{Q} \chi_{2}\left\langle\left(\sigma_{2}-\sigma_{1}\right)^{-1} \tilde{\eta}, \tilde{\eta}\right\rangle .
\end{aligned}
$$

Here $\tilde{\eta}=\tilde{\eta}(y)$ ranges over periodic vector fields, and the supremum is achieved when

$$
\chi_{2}\left(\tilde{\eta}-\left(\sigma_{2}-\sigma_{1}\right)(\xi+e(\phi))\right)=0 .
$$

One can get an inequality by making a special choice of $\tilde{\eta}$ in (2.6). We take $\tilde{\eta}(y) \equiv \eta$, where $\eta$ is any constant tensor. This gives

$$
\begin{aligned}
& \int_{Q}\left\langle\left(\sigma(y)-\sigma_{1}\right)(\xi+e(\phi)), \xi+e(\phi)\right\rangle \\
& \quad \geq 2 \theta_{2}\langle\eta, \xi\rangle-\theta_{2}\left\langle\left(\sigma_{2}-\sigma_{1}\right)^{-1} \eta, \eta\right\rangle+\int_{Q} 2 \chi_{2}\langle\eta, e(\phi)\rangle .
\end{aligned}
$$


Substitution into (2.5) yields the following, after a bit of simplification:

$$
\begin{aligned}
\left\langle\sigma^{*} \xi, \xi\right\rangle \geq & \left\langle\sigma_{1} \xi, \xi\right\rangle+2 \theta_{2}\langle\eta, \xi\rangle-\theta_{2}\left\langle\left(\sigma_{2}-\sigma_{1}\right)^{-1} \eta, \eta\right\rangle \\
& +\inf _{\phi} \int_{Q}\left[\left\langle\sigma_{1} e(\phi), e(\phi)\right\rangle+2 \chi_{2}\langle\eta, e(\phi)\rangle\right] .
\end{aligned}
$$

It remains to bound the infimum over $\phi$ in $(2.8)$; this is the "nonlocal term" referred to above. Let us rewrite it as

$$
\int_{Q}\left[\left|\sigma_{1}^{\frac{1}{2}} e(\phi)\right|^{2}+2 \chi_{2}\left\langle\sigma_{1}^{-\frac{1}{2}} \eta, \sigma_{1}^{\frac{1}{2}} e(\phi)\right\rangle\right],
$$

where $\sigma_{1}^{\frac{1}{2}}$ is the positive square root of $\sigma_{1}$, viewed as a symmetric linear map on the space $S$ of symmetric tensors. An application of Plancherel's formula shows that (2.9) is the same as

$$
\sum_{k \in \mathbf{Z}^{N}}\left[\left|\sigma_{1}^{\frac{1}{2}} \widehat{e(\phi)}(k)\right|^{2}+2 \operatorname{Re} \overline{\hat{\chi}_{2}(k)}\left\langle\sigma_{1}^{-\frac{1}{2}} \eta, \sigma_{1}^{\frac{1}{2}} \widehat{e(\phi)}(k)\right\rangle\right],
$$

where $\chi_{2}(y)=\sum \hat{\chi}_{2}(k) e^{2 \pi i k \cdot y}$, and $\langle\cdot, \cdot\rangle$ is the symmetric inner product on complex matrices. Choosing $\phi$ to minimize $(2.9)$ is the same as choosing $\hat{\phi}$ to minimize (2.10), which may be done separately at each $k$. Frequency 0 is special: it contributes nothing to $(2.10)$, since $\widehat{e(\phi)}(0)=0$ for any periodic $\phi$. When $k \neq 0$, the optimal value of $\gamma=\sigma_{1}^{\frac{1}{2}} \widehat{e(\phi)}(k)$ is obtained by minimizing

$$
|\gamma|^{2}+2 \operatorname{Re} \overline{\hat{\chi}_{2}(k)}\left\langle\sigma_{1}^{-\frac{1}{2}} \eta, \gamma\right\rangle
$$

over the space of all possible values of $\sigma_{1}^{\frac{1}{2}} \widehat{e(\phi)}(k)$, which is the complexification of $\sigma_{1}^{\frac{1}{2}} V(k)$. Some linear algebra shows that the optimal choice is

$$
\gamma=-\hat{\chi}_{2}(k) \pi_{\sigma_{1}^{1 / 2} V(k)}\left(\sigma_{1}^{-\frac{1}{2}} \eta\right)
$$

(see, e.g., [20, Lemma 3.2]), and substitution into (2.11) gives the value

$$
-\left|\hat{\chi}_{2}(k)\right|^{2}\left|\pi_{\sigma_{1}^{1 / 2} V(k)} \sigma_{1}^{-\frac{1}{2}} \eta\right|^{2} .
$$

We thus deduce this exact expression for the nonlocal term

$$
\inf _{\phi}(2.9)=-\sum_{k \neq 0}\left|\hat{\chi}_{2}(k)\right|^{2}\left|\pi_{\sigma_{1}^{1 / 2} V(k)} \sigma_{1}^{-\frac{1}{2}} \eta\right|^{2} .
$$

The desired bound is now quite easy. Since $V(k)$ depends only on $k /|k|$, it is immediate from (2.4) that

$$
\left|\pi_{\sigma_{1}^{\frac{1}{2}} V(k)} \sigma_{1}^{-\frac{1}{2}} \eta\right|^{2} \leq g(\eta) .
$$

An application of Plancherel's formula gives

$$
\sum_{k \neq 0}\left|\hat{\chi}_{2}(k)\right|^{2}=\int_{Q}\left|\chi_{2}(y)-\theta_{2}\right|^{2}=\theta_{1} \theta_{2} .
$$


Combining these two relations, we see that

$$
\inf _{\phi}(2.9) \geq-\theta_{1} \theta_{2} g(\eta)
$$

Returning to $(2.8)$, we have shown that

$$
\left\langle\sigma^{*} \xi, \xi\right\rangle \geq\left\langle\sigma_{1} \xi, \xi\right\rangle+2 \theta_{2}\langle\eta, \xi\rangle-\theta_{2}\left\langle\left(\sigma_{2}-\sigma_{1}\right)^{-1} \eta, \eta\right\rangle-\theta_{1} \theta_{2} g(\eta)
$$

for any (constant) symmetric tensors $\xi, \eta$. Maximizing the right-hand side with respect to $\eta$ yields the desired bound (2.3).

REMARK 2.2. A similar bound holds when $\sigma_{1}$ and $\sigma_{2}$ are only weakly wellordered, i.e., if $\sigma_{1} \leq \sigma_{2}$ but $\sigma_{2}-\sigma_{1}$ has a nontrivial null space. In that case, $\eta$ must be restricted to the range of $\sigma_{2}-\sigma_{1}$ in (2.3).

RemarK 2.3. Notice that $g(\eta)$ is convex, since (2.4) defines it as the maximum of a family of nonnegative quadratic forms. Thus the essential term in the lower bound,

$$
\sup _{\eta}\left[2\langle\xi, \eta\rangle-\left\langle\left(\sigma_{2}-\sigma_{1}\right)^{-1} \eta, \eta\right\rangle-\theta_{1} g(\eta)\right]
$$

is a concave maximization.

To evaluate this lower bound, it is obviously necessary to understand the function $g(\eta)$. This has been studied in the metallurgical literature, for a broad class of anisotropic $\sigma_{1}$; see [23] and the references cited there. (For a mathematical explanation of the significance of that literature, see [20].) The case when $\sigma_{1}$ is isotropic is treated in Sec. 7.

3. Attainability of the bound. In this section we show that the bound (2.3) is attainable. Specifically, for each value of $\xi$ we shall exhibit a composite (by describing the microstructure) for which (2.3) is an equality. It follows that (2.3) is an optimal bound, in the sense that there can be no better bound which depends only on $\xi, \theta_{1}$, $\theta_{2}, \sigma_{1}$, and $\sigma_{2}$.

Our method is that of sequential lamination. By now a familiar construction, this method has been used to establish the optimality of many different bounds; see, e.g., $[4,11,26,27,38]$. At the heart of our argument is a "layering formula" presented as Proposition 3.2. It was first established by Murat and Tartar in the context of conductivity [38] and by Francfort and Murat for elasticity [11]. Its close link to the Hashin-Shtrikman bound figures prominently in much of the recent literature on energy bounds $[4,5,22,24,25,29,38]$. The logic behind this linkage is understood by the experts, but is not explained clearly in the literature. We hope the treatment given here will appear less mysterious.

It may seem like cheating that we prove the bound for spatially periodic composites and then prove attainability using sequentially laminated ones. In fact, this is perfectly legitimate. Though sequentially laminated composites are not themselves spatially periodic, their effective tensors can be approximated arbitrarily well by ones associated with periodic microstructures. See Sec. 3 of [22] for a further discussion of this point and for a more expository treatment of sequential lamination.

The link between lamination formulas and the Hashin-Shtrikman principle arises, in essence, because the latter is exact for the special case of a (simply) laminated microstructure. This is the basis of the following result. 
Proposition 3.1. Let $\alpha$ and $\beta$ be two (possibly anisotropic) Hooke's laws, with $\alpha<\beta$. Let $\sigma^{*}$ be the effective Hooke's law of the composite obtained by layering $\alpha$ and $\beta$ in volume fractions $\theta_{\alpha}, \theta_{\beta}=1-\theta_{\alpha}$ respectively, in layers orthogonal to a unit vector $k$. Then $\sigma^{*}$ is determined by the formula

$$
\theta_{\beta}\left(\sigma^{*}-\alpha\right)^{-1}=(\beta-\alpha)^{-1}+\theta_{\alpha} f_{\alpha}(k),
$$

where $f_{\alpha}(k)$ is the (degenerate) Hooke's law associated to the quadratic form

$$
\left\langle f_{\alpha}(k) \xi, \xi\right\rangle=\left|\pi_{\alpha^{1 / 2} V(k)} \alpha^{-\frac{1}{2}} \xi\right|^{2} .
$$

Proof. This is equivalent to Theorem 4.1 of [11]; however, we shall give a new proof, parallel to that of Proposition 2.1, which clarifies the link between (2.4) and (3.2).

A layered composite can be considered spatially periodic, if we use a coordinate system such that $k$ is parallel to one axis. As usual, $\sigma^{*}$ is defined by

$$
\left\langle\sigma^{*} \xi, \xi\right\rangle=\inf _{\phi \text { per }} \int_{Q}\langle\sigma(y)[\xi+e(\phi)], \quad \xi+e(\phi)\rangle d y,
$$

where $\sigma(y)$ corresponds to the layered microstructure. It is well known that in this case the optimal $\phi=\phi^{\xi}$ is piecewise linear; in other words, the "strain" $\xi+e\left(\phi^{\xi}\right)$ is constant within each material.

Since $\alpha<\beta$, we are in the setting of the Hashin-Shtrikman variational principle. Let us re-examine (2.5)-(2.12) as they apply to the layered microgeometry. Since the optimal $\phi$ for (3.3) is piecewise linear, we have (for the layered microstructure only)

$$
\left\langle\sigma^{*} \xi, \xi\right\rangle=\inf _{\phi \in \mathrm{PCS}} \int_{Q}\langle\sigma(y)[\xi+e(\phi)], \xi+e(\phi)\rangle d y,
$$

in which PCS denotes the space of periodic displacement with constant strain in each of the layers. (PCS stands for "piecewise constant strain".) Arguing as for (2.6) gives

$$
\begin{aligned}
\left\langle\left(\sigma^{*}-\alpha\right) \xi, \xi\right\rangle=\inf _{\phi \in \mathrm{PCS}} \sup _{\eta}\left\{2 \theta_{\beta}\langle\eta, \xi\rangle-\theta_{\beta}\left\langle(\beta-\alpha)^{-1} \eta, \eta\right\rangle\right. & \\
& \left.\quad+\int_{Q}\left[2 \chi_{\beta}\langle\eta, e(\phi)\rangle+\langle\alpha e(\phi), e(\phi)\rangle\right] d y\right\},
\end{aligned}
$$

in which $\eta$ ranges over (constant) symmetric tensors. We may switch the inf and sup in (3.5) by applying a standard saddle point principle (see, e.g., [9]). This yields

$$
\begin{aligned}
& \left\langle\left(\sigma^{*}-\alpha\right) \xi, \xi\right\rangle=\sup _{\eta}\left\{2 \theta_{\beta}\langle\eta, \xi\rangle-\theta_{\beta}\left\langle(\beta-\alpha)^{-1} \eta, \eta\right\rangle\right. \\
& \left.\quad+\inf _{\phi \in \mathrm{PCS}} \int_{Q}\left[2 \chi_{\beta}\langle\eta, e(\phi)\rangle+\langle\alpha e(\phi), e(\phi)\rangle\right] d y\right\} .
\end{aligned}
$$

Because the microstructure is layered, the constraint of piecewise constant strain is redundant; the right-hand side of (3.6) is unchanged if we let $\phi$ range over all 
periodic displacement fields. The minimum over $\phi$ can be computed by Fourier transform, as in (2.9)-(2.12), leading to

$$
\inf _{\phi}=-\sum_{l \neq 0}\left|\hat{\chi}_{\beta}(l)\right|^{2}\left|\pi_{\alpha^{1 / 2} V(l)} \alpha^{-\frac{1}{2}} \eta\right|^{2} .
$$

Since the microstructure is layered, $\hat{\chi}_{\beta}(l)$ vanishes unless $l$ is parallel to $k$. Thus (3.7) becomes

$$
\begin{aligned}
\inf _{\phi} & =-\left(\sum_{l \neq 0}\left|\hat{\chi}_{\beta}(l)\right|^{2}\right)\left|\pi_{\alpha^{1 / 2} V(k)} \alpha^{-\frac{1}{2}} \eta\right|^{2} \\
& =-\theta_{\alpha} \theta_{\beta}\left|\pi_{\alpha^{1 / 2} V(k)} \alpha^{-\frac{1}{2}} \eta\right|^{2} .
\end{aligned}
$$

We thus have the exact formula

$$
\frac{1}{2}\left\langle\theta_{\beta}^{-1}\left(\sigma^{*}-\alpha\right) \xi, \xi\right\rangle=\sup _{\eta}\left[\langle\eta, \xi\rangle-\frac{1}{2}\left\langle(\beta-\alpha)^{-1} \eta, \eta\right\rangle-\frac{1}{2} \theta_{\alpha}\left\langle f_{\alpha}(k) \eta, \eta\right\rangle\right] .
$$

Taking the Legendre transform of each side yields (3.1).

The advantage of the layering formula (3.1) is that it is easy to iterate. Specifically, consider a family of "sequentially laminated composites" constructed as follows: $\sigma_{(0)}^{*}=\sigma_{2}, \sigma_{(1)}^{*}$ arises by layering $\sigma_{(0)}^{*}$ with $\sigma_{1}, \sigma_{(2)}^{*}$ arises by layering $\sigma_{(1)}^{*}$ with $\sigma_{1}$, and so forth. Iteration of (3.1) leads easily to formulas for $\left(\sigma_{(r)}^{*}-\sigma_{1}\right)^{-1}$, $r=1,2, \ldots$ After some bookkeeping, one deduces the following:

Proposition 3.2. Fix an integer $p \geq 1$; unit vectors $\left\{\nu_{i}\right\}_{i=1}^{p}$ in $\mathbf{R}^{N}$; real numbers $\left\{m_{i}\right\}_{i=1}^{p}$ with $0 \leq m_{i} \leq 1, \sum m_{i}=1$; and a real number $\theta_{1}, 0<\theta_{1}<1$. Let $\sigma_{1}$ and $\sigma_{2}$ be two Hooke's laws with $\sigma_{1}<\sigma_{2}$. Then there is a sequentially laminated composite made by mixing $\sigma_{1}$ and $\sigma_{2}$ in volume fractions $\theta_{1}$ and $\theta_{2}=1-\theta_{1}$, whose effective Hooke's law $\sigma^{*}$ is characterized by

$$
\theta_{2}\left(\sigma^{*}-\sigma_{1}\right)^{-1}=\left(\sigma_{2}-\sigma_{1}\right)^{-1}+\theta_{1} \sum_{i=1}^{p} m_{i} f_{\sigma_{1}}\left(\nu_{i}\right)
$$

We omit the proof since it is presented quite clearly in several places, including [22]. However, we note that the associated microstructures consist of plate-like inclusions of material 2, arranged appropriately in a matrix of material 1. If $p \geq 2$ then the sequentially laminated microstructure achieving (3.9) is not unique: different ways of labelling $\left\{\left(m_{i}, \nu_{i}\right)\right\}$ yield different microstructures. In particular, any of the vectors $\nu_{i}$ can be chosen as the normal vector determining the plane of the plate-like inclusions. (However, the inclusions must all be aligned.)

REMARK 3.3. The layering formulas (3.1) and (3.9) are actually valid for every pair of Hooke's laws $\sigma_{1}$ and $\sigma_{2}$, not necessarily well-ordered, provided only that $\sigma_{2}-\sigma_{1}$ is invertible. This observation, which will be crucial in [2], is proved in [11].

REMARK 3.4. If $\sigma_{1} \leq \sigma_{2}$ but $\sigma_{2}-\sigma_{1}$ has a nontrivial kernel, then $\sigma^{*}-\sigma_{1}$ has the same kernel and (3.9) holds when restricted to the image of $\sigma_{2}-\sigma_{1}$. This is easy to deduce from the above results by a perturbation argument. A similar assertion holds for any pair of Hooke's laws $\sigma_{1}$ and $\sigma_{2}$, not necessarily well-ordered; see [11] for this more general assertion.

We are now equipped to prove the optimality of the lower bound (2.2). 
Theorem 3.5. Assume that $\sigma_{1}<\sigma_{2}$. Then for any choice of $\xi$ and any volume fractions $\theta_{1}, \theta_{2}=1-\theta_{1}$, there is a sequentially laminated microstructure that achieves equality in (2.2).

Proof. Following [19, 22], we shall use the optimality condition for the maximization over $\eta$ in (2.3) to construct an extremal microstructure. The essence of the bound is the concave maximization

$$
\sup _{\eta}\left[2\langle\xi, \eta\rangle-\left\langle\left(\sigma_{2}-\sigma_{1}\right)^{-1} \eta, \eta\right\rangle-\theta_{1} g(\eta)\right]
$$

with $g$ defined by (2.4), or equivalently

$$
g(\eta)=\sup _{|\nu|=1}\left\langle f_{\sigma_{1}}(\nu) \eta, \eta\right\rangle .
$$

Since (3.10) is strictly concave, there is a unique extremal $\eta^{*}$.

The function $g$ is not smooth, so the appropriate tool for obtaining first-order optimality conditions is the subdifferential calculus or the calculus of generalized gradients; see, e.g., [7]. At the extremal $\eta^{*}$ we must have

$$
0 \in 2 \xi-2\left(\sigma_{2}-\sigma_{1}\right)^{-1} \eta^{*}-\theta_{1} \partial g\left(\eta^{*}\right),
$$

where $\partial g\left(\eta^{*}\right)$ is the subdifferential of $g$ at $\eta^{*}$. Since $g(\eta)$ is the supremum of a continuously parametrized family of nonnegative quadratic functions, its subdifferential is the convex hull of the gradients of those quadratic functions which are extremal. This gives

$$
\partial g\left(\eta^{*}\right)=\left\{\sum_{i=1}^{p} 2 m_{i} f_{\sigma_{1}}\left(\nu_{i}\right) \eta^{*}\right\},
$$

in which each $\nu_{i}$ must be extremal for (3.11) at $\eta=\eta^{*}, 0 \leq m_{i} \leq 1$, and $\sum m_{i}=1$. (We need only consider finite sums with at most $p=N(N+1) / 2+1$ elements, by Caratheodory's theorem.) Combining (3.12) and (3.13) gives the optimality condition

$$
\xi=\left(\sigma_{2}-\sigma_{1}\right)^{-1} \eta^{*}+\theta_{1} \sum m_{i} f_{\sigma_{1}}\left(\nu_{i}\right) \eta^{*}
$$

Taking the inner product with $\eta^{*}$ gives

$$
\left\langle\xi, \eta^{*}\right\rangle=\left\langle\left(\sigma_{2}-\sigma_{1}\right)^{-1} \eta^{*}, \eta^{*}\right\rangle+\theta_{1} g\left(\eta^{*}\right)
$$

therefore, the extremal value of $(3.10)$ is

$$
2\left\langle\xi, \eta^{*}\right\rangle-\left\langle\left(\sigma_{2}-\sigma_{1}\right)^{-1} \eta^{*}, \eta^{*}\right\rangle-\theta_{1} g\left(\eta^{*}\right)=\left\langle\xi, \eta^{*}\right\rangle,
$$

and the bound (2.3) can be expressed as

$$
\left\langle\sigma^{*} \xi, \xi\right\rangle \geq\left\langle\sigma_{1} \xi, \xi\right\rangle+\theta_{2}\left\langle\xi, \eta^{*}\right\rangle .
$$

To achieve equality, consider the composite provided by Proposition 3.2 with the parameters $m_{i}, \nu_{i}$ taken from the optimality condition (3.14). Comparing (3.9) with (3.14), we see that its effective Hooke's law $\sigma^{*}$ satisfies

$$
\theta_{2}\left(\sigma^{*}-\sigma_{1}\right)^{-1} \eta^{*}=\xi
$$


So for this composite

$$
\left\langle\left(\sigma^{*}-\sigma_{1}\right) \xi, \xi\right\rangle=\theta_{2}\left\langle\eta^{*}, \xi\right\rangle .
$$

In other words, this composite achieves equality in (3.16).

REMARK 3.6. If $\sigma_{1} \leq \sigma_{2}$ but $\sigma_{2}-\sigma_{1}$ has a nontrivial null-space, then the bound is still optimal. The proof proceeds as above but with $\eta$ restricted to the image of $\sigma_{2}-\sigma_{1}$. We make use here of Remarks 2.2 and 3.4.

REMARK 3.7. It is natural to ask what is the minimum rank of lamination $p_{\min }$ required for achieving the bound (2.3). The estimate given above, $p \leq$ $N(N+1) / 2+1$, is generally far from optimal. To evaluate $p_{\min }$, one must ask what is the dimension of the smallest linear space containing $\left\{f_{\sigma_{1}}\left(\nu_{i}\right) \eta^{*}\right\}$, where $\eta^{*}$ is extremal for (3.10), and $\left\{\nu_{i}\right\}$ are extremal for (3.11) at $\eta=\eta^{*}$. When $\sigma_{1}$ and $\sigma_{2}$ are isotropic, it turns out that $p_{\min } \leq N$ in space dimension $N$. In other words, rank$N$ lamination is sufficient to achieve the optimal lower bound on $\left\langle\sigma^{*} \xi, \xi\right\rangle$ in that case. The proof is parallel to that of Theorem 3.8 in [22]. Rank- $N$ lamination also suffices for achieving the optimal upper bound on $\left\langle\sigma^{*} \xi, \xi\right\rangle$ and the upper and lower bounds on complementary energy $\left\langle\left(\sigma^{*}\right)^{-1} \xi, \xi\right\rangle$ when $\sigma_{1}$ and $\sigma_{2}$ are isotropic.

Remark 3.8. The value of the optimal bound (2.3) is a $C^{1}$ function of $\xi$. Indeed, the right-hand side of $(2.3)$ is strictly concave in $\eta$, so the extremal $\eta$ is unique and it depends continuously on $\xi$. Let us denote it by $\eta[\xi]$. It is a standard result from convex analysis that in this circumstance

$$
\frac{\partial}{\partial \xi} \sup _{\eta}\left[2\langle\xi, \eta\rangle-\left\langle\left(\sigma_{2}-\sigma_{1}\right)^{-1} \eta, \eta\right\rangle-\theta_{1} g(\eta) \mid=2 \eta[\xi] .\right.
$$

So the gradient of the optimal energy with respect to $\xi$ is $2\left(\sigma_{1} \xi+\theta_{2} \eta[\xi]\right)$, which is continuous in $\xi$.

4. Alternative proof of the bound by the translation method. The "translation method" for bounding effective moduli is newer than the Hashin-Shtrikman variational principle. First applied by Murat and Tartar [37, 38] and by Lurie and Cherkaev [26], it has since been used successfully for many applications. The name "translation method" was introduced by Milton in his recent comprehensive study [28]. The basic idea is to "translate" the microscopic Hooke's law $\sigma(y)$ by a constant "quasi-convex" tensor $\tau$ and then to use an elementary bound (see Propositions 4.1 and 4.2). There are usually many plausible choices for $\tau$. This is at once the method's greatest strength (it is flexible) and its greatest weakness (one must choose $\tau$ correctly to get a good bound).

For a long time it seemed that the translation method and the Hashin-Shtrikman principle were unrelated. The link between them was found by Milton [28]. He showed that certain "trace bounds" originally derived using the Hashin-Shtrikman method can also be derived by translation. There are several practical reasons for exploring this connection further. First, it offers a link between translation bounds and the construction of sequentially laminated composites. Second, it displays a special class of translations that might be useful in other situations. Third, it provides a possible route for extending the Hashin-Shtrikman bounds to mixtures of non-wellordered components. 
Milton's treatment in [28] is somewhat complicated, because it refers to the general "trace bound". (That is equivalent to our bound on a sum of energies; see Sec. 5.) Here we present the specialization of Milton's argument to the bound on a single elastic energy. Besides providing some explication of Milton's work, this section lays the groundwork for our paper [2], which extends the optimal lower bound (2.3) to mixtures of two non-well-ordered isotropic materials.

We begin with a review of the translation method as it applies to linear elasticity. Recall the definition of $\sigma^{*}$ :

$$
\left\langle\sigma^{*} \xi, \xi\right\rangle=\inf _{\phi} \int_{Q}\langle\sigma(y)[\xi+e(\phi)], \xi+e(\phi)\rangle d y,
$$

where the infimum is over all $Q$-periodic $\phi$ and $e(\phi)=\frac{1}{2}\left(\nabla \phi+{ }^{t} \nabla \phi\right)$. There is a well-known lower bound, the "harmonic mean" or "Reuss" bound:

$$
\sigma^{*} \geq\left(\int_{Q} \sigma^{-1}(y) d y\right)^{-1}
$$

It arises from (4.1) by forgetting that $e=e(\phi)$ should come from a displacement and minimizing instead over the larger class of tensor fields $e=e(y)$ that satisfy only the "algebraic" constraint

$$
\int_{Q} e(y) d y=0
$$

To improve on (4.2), we should augment (4.3) by some additional algebraic relation satisfied by strains. This may be done as follows. Consider a constant fourth-order tensor $\tau$ with the symmetry of a Hooke's law. We call $\tau$ quasiconvex on strains if it satisfies

$$
\int_{Q}\langle\tau e(\phi), e(\phi)\rangle d y \geq 0
$$

for any $Q$-periodic $\phi$. Given any such tensor $\tau$, we have an associated lower bound on $\left\langle\sigma^{*} \xi, \xi\right\rangle$ :

$$
\left\langle\sigma^{*} \xi, \xi\right\rangle \geq \inf _{e(y)} \int_{Q}\langle\sigma(y)[\xi+e(y)], \xi+e(y)\rangle d y,
$$

in which $e(y)$ is constrained by (4.3) and also

$$
\int_{Q}\langle\tau e(y), e(y)\rangle \geq 0 \text {. }
$$

Clearly (4.5) is at least as good as (4.2), since the latter arises by omitting the constraint (4.6); however, (4.5) is not easy to evaluate. A more easily computed bound is obtained by considering an "augmented" variational principle, in which $\lambda \geq 0$ appears as a "Lagrange multiplier" for (4.6):

$$
\int_{Q}\langle\sigma(y)[\xi+e(y)], \quad \xi+e(y)\rangle d y-\lambda \int_{Q}\langle\tau e(y), e(y)\rangle,
$$

which is to be minimized now subject only to (4.3). There is no loss of generality in taking $\lambda=1$, since we can always replace $\tau$ by $\lambda \tau$. This leads to one version of the translation bound (cf. [28, (8.1)]): 
Proposition 4.1. Let $\sigma^{*}$ be the effective Hooke's law of a mixture of two or more elastic materials. Let $\tau$ be any constant fourth-order tensor which is quasiconvex on strains. Then

$$
\left\langle\sigma^{*} \xi, \xi\right\rangle \geq \inf _{e(y)}\left[\int_{Q}\langle\sigma(y)[\xi+e(y)], \xi+e(y)\rangle d y-\int_{Q}\langle\tau e(y), e(y)\rangle d y\right],
$$

in which $e(y)$ ranges over all symmetric second-order tensor fields with mean value zero.

Proof. The right-hand side of (4.8) is clearly smaller than the right-hand side of (4.5) if $e(y)$ satisfies (4.6). When this constraint is dropped, the right-hand side of (4.8) can only decrease.

A second version of the translation method, more explicit in character, obtained by evaluating the right-hand side of $(4.8)$ (cf. $[28,(7.7)]$ ) is:

Proposition 4.2. In the context of Proposition 4.1, suppose $\tau$ satisfies the additional "admissibility" constraint

$$
\sigma(y)-\tau \geq 0 \text { for every } y,
$$

in the sense that $\sigma(y)-\tau$ determines a nonnegative quadratic form. Then

$$
\sigma^{*} \geq\left(\int(\sigma(y)-\tau)^{-1} d y\right)^{-1}+\tau
$$

Proof. We rewrite (4.8) as

$$
\left\langle\left(\sigma^{*}-\tau\right) \xi, \xi\right\rangle \geq \inf _{e(y)} \int_{Q}\langle(\sigma(y)-\tau)[\xi+e(y)], \xi+e(y)\rangle d y,
$$

subject to $\int_{Q} e(y)=0$. Assume for the moment that

$$
\langle(\sigma(y)-\tau) \eta, \eta\rangle \geq \varepsilon|\eta|^{2}
$$

for some $\varepsilon>0$, independent of $y$. Then the right-hand side of (4.11) is strictly convex in $e(y)$, so it has a unique optimal $e^{*}(y)$, which satisfies

$$
(\sigma(y)-\tau)\left[\xi+e^{*}(y)\right]=\eta^{*}
$$

for some constant tensor $\eta^{*}$. We deduce from (4.13) that

$$
\xi=\int_{Q}(\sigma(y)-\tau)^{-1} \eta^{*} d y
$$

so

$$
\eta^{*}=\left(\int_{\Omega}(\sigma(y)-\tau)^{-1} d y\right)^{-1} \xi ;
$$

therefore, (4.11) yields

$$
\begin{aligned}
\left\langle\left(\sigma^{*}-\tau\right) \xi, \xi\right\rangle & \geq \int_{Q}\left\langle(\sigma(y)-\tau)\left[\xi+e^{*}(y)\right], \xi+e^{*}(y)\right\rangle \\
& =\int_{Q}\left\langle\eta^{*}, \xi+e^{*}(y)\right\rangle=\left\langle\eta^{*}, \xi\right\rangle .
\end{aligned}
$$


Thus

$$
\left\langle\left(\sigma^{*}-\tau\right) \xi, \xi\right\rangle \geq\left\langle\left(\int_{Q}(\sigma(y)-\tau)^{-1} d y\right)^{-1} \xi, \xi\right\rangle
$$

for every $\xi$. This is equivalent to (4.10).

If the inequality (4.9) is not strict, i.e., if (4.12) does not hold, we may argue as above with $\sigma(y)$ replaced by $\sigma(y)+\varepsilon I_{4}$, where $I_{4}$ is the fourth-order identity tensor. Passage to the limit $\varepsilon \rightarrow 0$ yields (4.10), provided that the right-hand side is interpreted appropriately.

REMARK 4.3. Note that "well-ordering" plays absolutely no role in the above. Also, the "admissibility condition" (4.9) is required only for Proposition 4.2, not for Proposition 4.1. If (4.9) fails, however, then the right-hand side of (4.8) will be $-\infty$ for some (and possibly all) choices of $\xi$.

REMARK 4.4. From (4.9) and (4.10) we have that $\sigma^{*}-\tau \geq 0$. So (4.10) can also be written in the equivalent form

$$
\left(\sigma^{*}-\tau\right)^{-1} \leq \int_{Q}(\sigma(y)-\tau)^{-1} d y
$$

To make use of these bounds, it is of course necessary that we be able to recognize when $\tau$ is quasiconvex on strains. The following lemma is familiar from the theory of quasiconvexity and compensated compactness $[11,31]$ and is in any case elementary.

LEMMA 4.5. Let $\tau$ by a constant fourth-order tensor with the symmetries of a Hooke's law. Then $\tau$ is quasiconvex on strains if and only if

$$
\langle\tau \xi, \xi\rangle \geq 0 \text { for } \xi \in V(k)
$$

for every $k \neq 0$. Here $V(k)$ is defined by (2.2).

Proof. If $\tau$ satisfies (4.15) then (4.4) holds, by an application of Plancherel's theorem. Conversely, if (4.15) fails for some $\xi_{0} \in V\left(k_{0}\right)$, then one easily constructs a counterexample to (4.4) by taking $\phi$ to be a suitable function of $y \cdot k_{0}$. (We may suppose without loss of generality that $k_{0}$ has integer components, so $\phi$ can be taken to be $Q$-periodic.)

We turn now to the task of recovering the Hashin-Shtrikman lower bound (2.3) by the translation method. The key is to consider the special translations $\tau=\tau_{\eta}$ defined by

$$
\left\langle\tau_{\eta} \xi, \xi\right\rangle=\left\langle\sigma_{1} \xi, \xi\right\rangle-g(\eta)^{-1}\langle\eta, \xi\rangle^{2},
$$

where $\eta$ is any symmetric second-order tensor and $g(\eta)$ is defined by (2.4).

LEMMA 4.6. The translation $\tau_{\eta}$ is quasiconvex on strains.

Proof. We note that $g(\eta)>0$ if $\eta \neq 0$, so (4.16) makes sense. Indeed, if $g(\eta)$ were zero then $\sigma_{1}^{-\frac{1}{2}} \eta$ would be orthogonal to $\sigma_{1}^{\frac{1}{2}} V(k)$ for every $k$; but then $\langle\eta, \xi\rangle=0$ for every $\xi \in V(k)$ and every $k$. When $\xi=\nu \otimes k+k \otimes \nu$, this says $\langle\eta k, \nu\rangle=0$ for all $\nu$, so $\eta k=0$. As $k$ varies, this forces $\eta=0$. 
To prove quasi-convexity on strains, we need only verify (4.15). If $k \neq 0$ and $\xi \in V(k)$, then

$$
\begin{aligned}
\langle\xi, \eta\rangle^{2} & =\left\langle\sigma_{1}^{\frac{1}{2}} \xi, \sigma_{1}^{-\frac{1}{2}} \eta\right\rangle^{2} \\
& =\left\langle\sigma_{1}^{\frac{1}{2}} \xi, \pi_{\sigma_{1}^{1 / 2} V(k)} \sigma_{1}^{-\frac{1}{2}} \eta\right\rangle^{2} \\
& \leq\left|\sigma_{1}^{\frac{1}{2}} \xi\right|^{2} \cdot\left|\pi_{\sigma_{1}^{1 / 2} V(k)} \sigma_{1}^{-\frac{1}{2}} \eta\right|^{2} \\
& \leq\left\langle\sigma_{1} \xi, \xi\right\rangle \cdot g(\eta) .
\end{aligned}
$$

It follows that

$$
\left\langle\tau_{\eta} \xi, \xi\right\rangle=\left\langle\sigma_{1} \xi, \xi\right\rangle-g(\eta)^{-1}\langle\eta, \xi\rangle^{2} \geq 0
$$

as desired.

Lemma 4.7. Consider a composite made from two materials $\sigma_{1}$ and $\sigma_{2}$ which are well-ordered, i.e., such that $\sigma_{1} \leq \sigma_{2}$. Then $\tau_{\eta}$ is admissible in the sense of (4.9).

Proof. We need only check that $\sigma_{1} \geq \tau_{\eta}$ and $\sigma_{2} \geq \tau_{\eta}$. The first relation is true since

$$
\left\langle\sigma_{1} \xi, \xi\right\rangle-\left\langle\tau_{\eta} \xi, \xi\right\rangle=g(\eta)^{-1}\langle\eta, \xi\rangle^{2} \geq 0 .
$$

The second follows from the first, since $\sigma_{2} \geq \sigma_{1}$.

We have given two slightly different formulations of the translation bound, in formulas (4.10) and (4.14). There are correspondingly two different approaches to recovering (2.3). We shall present them both, in part to highlight the fact that they are not quite the same.

Our first approach is based on (4.14). It suffices to show that

$$
\left\langle\sigma^{*} \xi, \xi\right\rangle \geq\left\langle\sigma_{1} \xi, \xi\right\rangle+\theta_{2}\left[2\langle\xi, \eta\rangle-\left\langle\left(\sigma_{2}-\sigma_{1}\right)^{-1} \eta, \eta\right\rangle-\theta_{1} g(\eta)\right]
$$

for any (constant) $\xi$ and $\eta$, since $(2.3)$ is then obtained by optimization over $\eta$.

Proposition 4.8. Assume that $\sigma_{1}<\sigma_{2}$. For any constant tensor $\eta$, consider the translation $\tau_{\eta}$, defined by (4.16), and the associated "translation bound"

$$
\left(\sigma^{*}-\tau_{\eta}\right)^{-1} \leq \int_{Q}\left(\sigma(y)-\tau_{\eta}\right)^{-1} d y .
$$

When evaluated at $\eta,(4.18)$ becomes

$$
\theta_{2}\left\langle\left(\sigma^{*}-\sigma_{1}\right)^{-1} \eta, \eta\right\rangle \leq\left\langle\left(\sigma_{2}-\sigma_{1}\right)^{-1} \eta, \eta\right\rangle+\theta_{1} g(\eta) .
$$

This relation is equivalent to the validity of (4.17) for every $\xi$, with $\eta$ held fixed.

Proof. We may write (4.16) in the form $\tau_{\eta}=\sigma_{1}-g(\eta)^{-1} \eta \otimes \eta$. In this notation

$$
\begin{aligned}
& \sigma_{1}-\tau_{\eta}=g(\eta)^{-1} \eta \otimes \eta, \\
& \sigma_{2}-\tau_{\eta}=\left(\sigma_{2}-\sigma_{1}\right)+g(\eta)^{-1} \eta \otimes \eta .
\end{aligned}
$$

Now recall the following elementary fact of linear algebra: if $D$ is a symmetric invertible matrix, $c$ a real number, and $u$ a vector, then $H=D+c u \otimes u$ is invertible and

$$
H^{-1}=D^{-1}-\frac{c}{1+c\left\langle u, D^{-1} u\right\rangle} D^{-1}(u \otimes u) D^{-1} .
$$


In particular, contracting with $u \otimes u$,

$$
c\left\langle H^{-1} u, u\right\rangle=1-\frac{1}{1+c\left\langle u, D^{-1} u\right\rangle} .
$$

If $D$ is singular one can apply the above to $D+\varepsilon I$ and pass to the limit $\varepsilon \rightarrow 0$.

When evaluated at $\eta$, the translation bound (4.18) becomes

$$
\left\langle\left(\sigma^{*}-\tau_{\eta}\right)^{-1} \eta, \eta\right\rangle \leq \theta_{1}\left\langle\left(\sigma_{1}-\tau_{\eta}\right)^{-1} \eta, \eta\right\rangle+\theta_{2}\left\langle\left(\sigma_{2}-\tau_{\eta}\right)^{-1} \eta, \eta\right\rangle .
$$

Several applications of (4.21) give

$$
\begin{aligned}
& g(\eta)^{-1}\left\langle\left(\sigma^{*}-\tau_{\eta}\right)^{-1} \eta, \eta\right\rangle=1-\frac{1}{1+g(\eta)^{-1}\left\langle\left(\sigma^{*}-\sigma_{1}\right)^{-1} \eta, \eta\right\rangle} \\
& g(\eta)^{-1}\left\langle\left(\sigma_{2}-\tau_{\eta}\right)^{-1} \eta, \eta\right\rangle=1-\frac{1}{1+g(\eta)^{-1}\left\langle\left(\sigma_{2}-\sigma_{1}\right)^{-1} \eta, \eta\right\rangle} \\
& g(\eta)^{-1}\left\langle\left(\sigma_{1}-\tau_{\eta}\right)^{-1} \eta, \eta\right\rangle=1-\frac{1}{\infty}=1 .
\end{aligned}
$$

Thus (4.22) is equivalent to

$$
\frac{1}{1+g(\eta)^{-1}\left\langle\left(\sigma^{*}-\sigma_{1}\right)^{-1} \eta, \eta\right\rangle} \geq \frac{\theta_{2}}{1+g(\eta)^{-1}\left\langle\left(\sigma_{2}-\sigma_{1}\right)^{-1} \eta, \eta\right\rangle},
$$

which leads after elementary manipulation to (4.19).

Now let us verify that (4.19) is equivalent to the validity of (4.17) for every $\xi$. One can rewrite $(4.17)$ as

$$
2 \theta_{2}\langle\xi, \eta\rangle-\left\langle\left(\sigma^{*}-\sigma_{1}\right) \xi, \xi\right\rangle \leq \theta_{2}\left[\left\langle\left(\sigma_{2}-\sigma_{1}\right)^{-1} \eta, \eta\right\rangle+\theta_{1} g(\eta)\right] .
$$

We know $\sigma^{*}>\sigma_{1}$ as a consequence of the harmonic mean bound and the hypothesis that $\sigma_{2}>\sigma_{1}$, so the maximum of the left-hand side of (4.23) is achieved when $\theta_{2} \eta=\left(\sigma^{*}-\sigma_{1}\right) \xi$, and its value is $\theta_{2}^{2}\left\langle\eta,\left(\sigma^{*}-\sigma_{1}\right)^{-1} \eta\right\rangle$. Thus (4.23) holds for all $\xi$ if and only if

$$
\theta_{2}^{2}\left\langle\eta,\left(\sigma^{*}-\sigma_{1}\right)^{-1} \eta\right\rangle \leq \theta_{2}\left[\left\langle\left(\sigma_{2}-\sigma_{1}\right)^{-1} \eta, \eta\right\rangle+\theta_{1} g(\eta)\right] .
$$

This is the same as (4.19).

Our second approach to recovering (2.3) makes use of (4.10) instead of (4.14). It is this approach that generalizes to the non-well-ordered case; see [2]. Actually, (4.10) seems to us the more natural starting point, since we are seeking a lower bound on $\left\langle\sigma^{*} \xi, \xi\right\rangle$. Though it leads once again to the optimal bound (2.3), it does not arrive there via (4.17).

Proposition 4.9. When evaluated at $\xi$ using the translation $\tau_{\eta},(4.10)$ asserts that

$$
\left\langle\sigma^{*} \xi, \xi\right\rangle \geq\left\langle\sigma_{1} \xi, \xi\right\rangle+\frac{\theta_{2}\langle\eta, \xi\rangle^{2}}{\theta_{1} g(\eta)+\left\langle\left(\sigma_{2}-\sigma_{1}\right)^{-1} \eta, \eta\right\rangle} .
$$

After optimization over $\eta,(4.24)$ is equivalent to the optimal bound (2.3). 
Proof. Recall that (4.10) was derived from the variational principle (4.8). So our goal is to calculate

$$
\inf _{e(y)} \int_{Q}\left[\langle\sigma(y)(\xi+e(y)), \quad \xi+e(y)\rangle-\left\langle\tau_{\eta} e(y), e(y)\right\rangle\right] d y,
$$

where $e(y)$ ranges over $Q$-periodic symmetric tensor fields with mean value zero. From the proof of Proposition 4.2, it is clear that an optimal $e^{*}(y)$ exists which is piecewise constant. If $e^{*}=e_{i}$ in material $i$, then the optimality condition for (4.25) is

$$
\begin{aligned}
g^{-1}(\eta)\left\langle\eta, \xi+e_{1}\right\rangle \eta & =c, \\
{\left[\left(\sigma_{2}-\sigma_{1}\right)+g(\eta)^{-1} \eta \otimes \eta\right]\left(\xi+e_{2}\right) } & =c
\end{aligned}
$$

for some constant second-order tensor $c$. Using (4.20), we deduce from (4.26) that

$$
\xi+e_{2}=\frac{\left\langle\eta, \xi+e_{1}\right\rangle}{g(\eta)+\left\langle\left(\sigma_{2}-\sigma_{1}\right)^{-1} \eta, \eta\right\rangle}\left(\sigma_{2}-\sigma_{1}\right)^{-1} \eta .
$$

Since $\theta_{1} e_{1}+\theta_{2} e_{2}=0$, we get from (4.27) that

$$
\left\langle\eta, e_{1}\right\rangle=\frac{\theta_{2}\langle\eta, \xi\rangle}{\theta_{1}+g(\eta)^{-1}\left\langle\left(\sigma_{2}-\sigma_{1}\right)^{-1} \eta, \eta\right\rangle} .
$$

Substitution into (4.25) yields

$$
\begin{aligned}
\int_{Q}\left[\left\langle\sigma(y)\left(\xi+e^{*}\right), \xi+e^{*}\right\rangle-\left\langle\tau_{\eta} e^{*}, e^{*}\right\rangle\right] d y & =\left\langle\tau_{\eta} \xi, \xi\right\rangle+\langle\xi, c\rangle \\
& =\left\langle\sigma_{1} \xi, \xi\right\rangle+g(\eta)^{-1}\langle\eta, \xi\rangle\left\langle\eta, e_{1}\right\rangle .
\end{aligned}
$$

Combining this with (4.28) gives (4.24).

In general, (4.24) is not at all the same as (4.17); however, we assert that the two coincide after optimization in $\eta$. To prove this, we must show that

$$
\left\langle\sigma^{*} \xi, \xi\right\rangle \geq\left\langle\sigma_{1} \xi, \xi\right\rangle+\sup _{\eta} \frac{\theta_{2}\langle\eta, \xi\rangle^{2}}{\theta_{1} g(\eta)+\left\langle\left(\sigma_{2}-\sigma_{1}\right)^{-1} \eta, \eta\right\rangle}
$$

is equivalent to (2.3). One proof is based on the observation that (2.3) and (4.29) have the same optimality condition (3.12). A less direct but more elementary argument proceeds as follows. Let $\eta^{*}$ be extremal for (2.3). We know from (3.15) that

$$
\left\langle\xi, \eta^{*}\right\rangle=\theta_{1} g\left(\eta^{*}\right)+\left\langle\left(\sigma_{2}-\sigma_{1}\right)^{-1} \eta^{*}, \eta^{*}\right\rangle .
$$

When evaluated at $\eta=\eta^{*},(4.24)$ becomes

$$
\left\langle\sigma^{*} \xi, \xi\right\rangle \geq\left\langle\sigma_{1} \xi, \xi\right\rangle+\theta_{2}\left\langle\xi, \eta^{*}\right\rangle .
$$

We proved in Sec. 3 that no better bound is possible, i.e., that there is a microstructure achieving equality in (4.31). (Note that (4.31) is the same as (3.16).) So $\eta^{*}$ must achieve the optimum in (4.29) as well, and (4.29) is equivalent to (2.3). 
5. Sums of energies. We explained in Sec. 2 and 3 how the Hashin-Shtrikman variational principle gives the optimal lower bound on $\left\langle\sigma^{*} \xi, \xi\right\rangle$ as the extremal value of a concave maximization. The same approach also works for sums of energies. We sketch that extension here.

As usual, we consider composites $\sigma^{*}$ made from two well-ordered, possibly anisotropic, materials $\sigma_{1}$ and $\sigma_{2}$, in volume fractions $\theta_{1}$ and $\theta_{2}$ respectively. Our goal is an optimal lower bound of the form

$$
\left\langle\sigma^{*} \xi_{1}, \xi_{1}\right\rangle+\cdots+\left\langle\sigma^{*} \xi_{J}, \xi_{J}\right\rangle \geq f_{-}\left(\xi_{1}, \ldots, \xi_{J}\right),
$$

where $\xi_{1}, \ldots, \xi_{J}$ are an arbitrary family of $J$ second-order symmetric tensors.

The analogue of (2.3) is (5.1) with

$$
f_{-}=\sum_{j=1}^{J}\left\langle\sigma_{1} \xi_{j}, \xi_{j}\right\rangle+\theta_{2} \sup \left[\sum_{j=1}^{J}\left\{2\left\langle\xi_{j}, \eta_{j}\right\rangle-\left\langle\left(\sigma_{2}-\sigma_{1}\right)^{-1} \eta_{j}, \eta_{j}\right\rangle\right\}-\theta_{1} g\left(\eta_{1}, \ldots, \eta_{J}\right)\right] \text {. }
$$

Here the supremum is over $\eta_{1}, \ldots, \eta_{J}$ and $g\left(\eta_{1}, \ldots, \eta_{J}\right)$ is defined by

$$
g\left(\eta_{1}, \ldots, \eta_{J}\right)=\sup _{|k|=1} \sum_{j=1}^{J}\left|\pi_{\sigma_{1}^{1 / 2} V(k)} \sigma_{1}^{-\frac{1}{2}} \eta_{j}\right|^{2}
$$

for any second-order symmetric tensors $\eta_{1}, \ldots, \eta_{J}$.

The proof of (5.2) follows that of Proposition 2.1. Indeed, (2.8) and (2.12) yield

$$
\begin{aligned}
\left\langle\sigma^{*} \xi_{j}, \xi_{j}\right\rangle \geq & \left\langle\sigma_{1} \xi_{j}, \xi_{j}\right\rangle+2 \theta_{2}\left\langle\eta_{j}, \xi_{j}\right\rangle-\theta_{2}\left\langle\left(\sigma_{2}-\sigma_{1}\right)^{-1} \eta_{j}, \eta_{j}\right\rangle \\
& +\sum_{k \neq 0}\left|\hat{\chi}_{2}(k)\right|^{2}\left|\pi_{\sigma_{1}^{1 / 2} V(k)} \sigma_{1}^{-\frac{1}{2}} \eta_{j}\right|^{2}
\end{aligned}
$$

for each $j=1, \ldots, J$. Addition of these relations leads easily to (5.2).

The proof that (5.2) is optimal is much the same as for a single energy. The optimality condition is

$$
0 \in 2 \vec{\xi}-2\left(\sigma_{2}-\sigma_{1}\right)^{-1} \vec{\eta}^{*}-\theta_{1} \partial g(\vec{\eta}),
$$

where we view $\vec{\xi}=\left(\xi_{1}, \ldots, \xi_{J}\right)$ as a vector whose components are symmetric tensors (cf. (3.12)). The subdifferential of $g$ is

$$
\partial g\left(\vec{\eta}^{*}\right)=\left\{\sum_{i=1}^{p} m_{i} \cdot 2 f_{\sigma_{1}}\left(\nu_{i}\right) \vec{\eta}^{*}\right\}
$$

in which each $\nu_{i}$ is extremal for (5.3) at $\vec{\eta}=\vec{\eta}^{*}, 0 \leq m_{i} \leq 1$, and $\sum m_{i}=1$ (cf. (3.13)). Arguing as in (3.15)-(3.17), one verifies that the sequentially laminated composite associated to these $\left\{\left(m_{i}, \nu_{i}\right)\right\}$ achieves equality in (5.2).

In space dimension $N$, the vector space of symmetric second-order tensors has dimension $N(N+1) / 2$. As a result, there is never any reason to consider $J>$ $N(N+1) / 2$ in (5.2). Indeed, we may write

$$
\sum_{j=1}^{J}\left\langle\sigma^{*} \xi_{j}, \xi_{j}\right\rangle=\left\langle\sigma^{*}, M\right\rangle
$$


where $M$ is the fourth-order tensor

$$
M=\sum_{j=1}^{J} \xi_{j} \otimes \xi_{j} .
$$

Clearly $M$ has the symmetries of a Hooke's law, and the associated quadratic form on symmetric second-order tensors is nonnegative. The most general such $M$ has a decomposition of the form (5.8) with $J=N(N+1) / 2$.

Our bound (5.2) is equivalent to the "trace bound" discussed in [28, 29]. To see this, observe that $(5.1)-(5.2)$ is equivalent to the assertion that

$$
\begin{aligned}
& \sum_{j=1}^{J}\left[2\left\langle\xi_{j}, \eta_{j}\right\rangle-\frac{1}{\theta_{2}}\left\langle\left(\sigma^{*}-\sigma_{1}\right) \xi_{j}, \xi_{j}\right\rangle\right] \\
& \quad \leq \sum_{j=1}^{J}\left\langle\left(\sigma_{2}-\sigma_{1}\right)^{-1} \eta_{j}, \eta_{j}\right\rangle+\theta_{1} g\left(\eta_{1}, \ldots, \eta_{J}\right)
\end{aligned}
$$

for every $\xi_{1}, \ldots, \xi_{J}$ and $\eta_{1}, \ldots, \eta_{J}$. Optimizing over $\xi_{j},(5.9)$ is equivalent to

$$
\theta_{2} \sum_{j=1}^{J}\left\langle\left(\sigma^{*}-\sigma_{1}\right)^{-1} \eta_{j}, \eta_{j}\right\rangle \leq \sum_{j=1}^{J}\left\langle\left(\sigma_{2}-\sigma_{1}\right)^{-1} \eta_{j}, \eta_{j}\right\rangle+\theta_{1} g\left(\eta_{1}, \ldots, \eta_{J}\right)
$$

for every $\eta_{1}, \ldots, \eta_{J}$. Writing $M=\sum \eta_{j} \otimes \eta_{j}$, this may be rewritten as

$$
\theta_{2}\left\langle\left(\sigma^{*}-\sigma_{1}\right)^{-1}, M\right\rangle \leq\left\langle\left(\sigma_{2}-\sigma_{1}\right)^{-1}, M\right\rangle+\theta_{1} g(M)
$$

for every nonnegative Hooke's law $M$, with

$$
g(M)=\sup _{|k|=1}\left\langle f_{\sigma_{1}}(k), M\right\rangle .
$$

This is precisely the "trace bound"; cf. [29, (6.62)] or [28, (6.11)].

6. Upper bounds and complementary energy. We have thus far focussed exclusively on lower rather than upper bounds and on elastic rather than complementary energy. Similar arguments work, however, for upper bounds and for complementary energy. We present the resulting optimal bounds in this section.

Upper bounds on elastic energy. As is well known, to get an upper bound on $\left\langle\sigma^{*} \xi, \xi\right\rangle$ one should use $\sigma_{2}$ rather than $\sigma_{1}$ as the "reference material" in the HashinShtrikman variational principle. The resulting analogue of $(2.3)$ is

$$
\left\langle\sigma^{*} \xi, \xi\right\rangle \leq\left\langle\sigma_{2} \xi, \xi\right\rangle+\theta_{1} \inf _{\eta}\left[2\langle\xi, \eta\rangle+\left\langle\left(\sigma_{2}-\sigma_{1}\right)^{-1} \eta, \eta\right\rangle-\theta_{2} h(\eta)\right],
$$

where $h(\eta)$ is defined by

$$
h(\eta)=\inf _{|k|=1}\left|\pi_{\sigma_{2}^{1 / 2} V(k)} \sigma_{2}^{-\frac{1}{2}} \eta\right|^{2} .
$$


The proof is almost entirely parallel to that of (2.3). The main difference is that (2.6) is replaced by

$$
\begin{aligned}
\int_{Q}\left\langle\left(\sigma(y)-\sigma_{2}\right)(\xi+e(\phi)), \xi+e(\phi)\right\rangle \\
\quad=\int_{Q} \chi_{1}\left\langle\left(\sigma_{1}-\sigma_{2}\right)(\xi+e(\phi)), \xi+e(\phi)\right\rangle \\
\quad=\inf _{\tilde{\eta}(y)} \int_{Q}-2 \chi_{1}\langle\tilde{\eta}, \xi+e(\phi)\rangle-\int_{Q} \chi_{1}\left\langle\left(\sigma_{1}-\sigma_{2}\right)^{-1} \tilde{\eta}, \tilde{\eta}\right\rangle .
\end{aligned}
$$

The right-hand side of (6.1) involves a convex minimization, since

$$
\left\langle\left(\sigma_{2}-\sigma_{1}\right)^{-1} \eta, \eta\right\rangle-\theta_{2} h(\eta)
$$

is a convex function of $\eta$. This is not immediately obvious. However, we can rewrite (6.3) as

$$
\sup _{|k|=1}\left[\left\langle\left(\sigma_{2}-\sigma_{1}\right)^{-1} \eta, \eta\right\rangle-\theta_{2}\left|\pi_{\sigma_{2}^{1 / 2} V(k)} \sigma_{2}^{-\frac{1}{2}} \eta\right|^{2}\right] .
$$

We are assuming that $\sigma_{1}<\sigma_{2}$, so

$$
\left\langle\left(\sigma_{2}-\sigma_{1}\right)^{-1} \eta, \eta\right\rangle \geq\left\langle\sigma_{2}^{-1} \eta, \eta\right\rangle=\left|\sigma_{2}^{-\frac{1}{2}} \eta\right|^{2} \geq\left|\pi_{\sigma_{2}^{1 / 2} V(k)} \sigma_{2}^{-\frac{1}{2}} \eta\right|^{2} .
$$

Thus (6.4) expresses (6.3) as a supremum of nonnegative quadratic forms in $\eta$, from which convexity follows.

The proof that (6.1) is optimal parallels the argument in Sec. 3. It uses the analogue of Proposition 3.2 with the roles of $\sigma_{1}$ and $\sigma_{2}$ reversed. In other words, the sequentially laminated composites of interest satisfy

$$
\theta_{1}\left(\sigma^{*}-\sigma_{2}\right)^{-1}=\left(\sigma_{1}-\sigma_{2}\right)^{-1}+\theta_{2} \sum_{i=1}^{p} m_{i} f_{\sigma_{2}}\left(\nu_{i}\right)
$$

instead of (3.9). The associated microstructures consist of plate-like inclusions of material 1 , arranged appropriately in a matrix of material 2 .

It is natural to ask whether there is an alternative proof of (6.1) based on translation, analogous to the proof of (2.3) presented in Sec. 4. The answer seems to be no. The difficulty is that as presented in Sec. 4, the translation method yields only lower bounds (see, e.g., (4.10)). There is an indirect way to proceed, however. We shall show that the optimal lower bound on complementary energy, formula (6.9), can be proved using translation. We shall also show that (6.9) leads to (6.1) by algebraic manipulation (see (6.18)-(6.21)). These two facts in combination yield a (somewhat indirect) proof of $(6.1)$ by translation.

There is of course an extension of (6.1) to sums of energies. Its formulation is left to the reader.

Bounds on complementary energy. Our arguments have thus far been based on (1.9), the variational characterization of the "elastic energy" quadratic form $\left\langle\sigma^{*} \xi, \xi\right\rangle$. They have led us to optimal upper and lower bounds on $\left\langle\sigma^{*} \xi, \xi\right\rangle$. There is also a well-known variational characterization of the "complementary energy" quadratic 
form:

$$
\left\langle\left(\sigma^{*}\right)^{-1} \xi, \xi\right\rangle=\inf _{\substack{\operatorname{div} \zeta=0 \\ \int \zeta=\xi}} \int_{Q}\left\langle\sigma(y)^{-1} \zeta, \zeta\right\rangle d y .
$$

Here $\zeta$ ranges over $Q$-periodic second-order symmetric tensor fields with divergence zero and mean value $\xi$; see, e.g., [36]. By using (6.6) in place of (1.9), we shall get optimal upper and lower bounds on the complementary energy $\left\langle\left(\sigma^{*}\right)^{-1} \xi, \xi\right\rangle$.

We must introduce some notation before proceeding. For $k \in \mathbf{R}^{N}, k \neq 0$, we define

$$
W(k)=\{\xi \in S: \xi \cdot k=0\},
$$

where $S$ is the space of all $N \times N$ symmetric second-order tensors. The subspace $W(k)$ will play a role below analogous to that of $V(k)$ in Sec. 2-4. Notice that $W(k)$ is actually the orthogonal complement of $V(k)$ in $S$. Moreover, for any Hooke's law $\sigma$ and any $k \neq 0, S$ has the orthogonal decomposition

$$
S=\sigma^{\frac{1}{2}} V(k) \oplus \sigma^{-\frac{1}{2}} W(k) .
$$

We turn now to the lower bound on complementary energy, which is the analogue of (2.3) based on (6.6) instead of (1.9). It asserts that

$$
\left\langle\left(\sigma^{*}\right)^{-1} \xi, \xi\right\rangle \geq\left\langle\sigma_{2}^{-1} \xi, \xi\right\rangle+\theta_{1} \sup _{\eta}\left[2\langle\xi, \eta\rangle-\left\langle\left(\sigma_{1}^{-1}-\sigma_{2}^{-1}\right)^{-1} \eta, \eta\right\rangle-\theta_{2} g_{c}(\eta)\right],
$$

with

$$
g_{\mathrm{c}}(\eta)=\sup _{|k|=1}\left|\pi_{\sigma_{2}^{-1 / 2} W(k)} \sigma_{2}^{\frac{1}{2}} \eta\right|^{2} .
$$

Our notation is designed to emphasize that $g_{c}$ is the analogue of $g$ (defined by (2.4)) for complementary energy.

The proof of (6.9) is parallel to that of (2.3), using $\sigma_{2}$ as the "reference material". That is the appropriate choice because $\sigma_{2}^{-1}<\sigma_{1}^{-1}$.

The proof that (6.9) is optimal proceeds as in Sec. 3. The key ingredient is the following complementary energy analogue of (3.9): there exists a composite $\sigma^{*}$ made from $\sigma_{1}$ and $\sigma_{2}$ in volume fractions $\theta_{1}$ and $\theta_{2}$ such that

$$
\theta_{1}\left(\left(\sigma^{*}\right)^{-1}-\sigma_{2}^{-1}\right)^{-1}=\left(\sigma_{1}^{-1}-\sigma_{2}^{-1}\right)^{-1}+\theta_{2} \sum_{i=1}^{p} m_{i} f_{\sigma_{2}}^{\mathrm{c}}\left(V_{i}\right),
$$

where $f_{\sigma_{2}}^{\mathrm{c}}(\nu)$ is the Hooke's law associated with the quadratic form

$$
\left\langle f_{\sigma_{2}}^{\mathrm{c}}(\nu) \eta, \eta\right\rangle=\left|\pi_{\sigma_{2}^{-1 / 2} W(k)} \sigma_{2}^{\frac{1}{2}} \eta\right|^{2} .
$$

The proof of (6.11) is parallel to that of Proposition 3.2, using a complementary energy version of the layering formula in place of Proposition 3.1. The microstructures associated to (6.11) consist of platelike inclusions of material 1 in a matrix of material 2.

The bound (6.9) can also be proved using the translation method. The discussion of Sec. 4 carries over straightforwardly to complementary energy. The only difference 
is in the notion of quasiconvexity. Instead of (4.4), the appropriate condition is that $\tau$ be quasiconvex on stresses, i.e.,

$$
\int_{Q}\langle\tau \zeta, \zeta\rangle d y \geq 0
$$

for any $Q$ periodic symmetric second-order tensor field $\zeta$ with $\operatorname{div} \zeta=0$ and $\int_{Q} \zeta=0$. The analogue of Lemma 4.5 is the observation that $\tau$ is quasiconvex on stresses if and only if

$$
\langle\tau \xi, \xi\rangle \geq 0 \text { for } \xi \in W(k), \text { for all } k \neq 0 .
$$

The appropriate translations for recovering (6.9) are naturally

$$
\left\langle\tau_{\eta} \xi, \xi\right\rangle=\left\langle\sigma_{2}^{-1} \xi, \xi\right\rangle-g_{\mathrm{c}}(\eta)^{-1}\langle\eta, \xi\rangle^{2},
$$

the analogue of (4.16) for complementary energy. The details can safely be left to the reader.

We turn finally to the upper bound on complementary energy, which is the analogue of $(6.1)$ for complementary energy:

$$
\left\langle\left(\sigma^{*}\right)^{-1} \xi, \xi\right\rangle \leq\left\langle\sigma_{1}^{-1} \xi, \xi\right\rangle+\theta_{2} \inf _{\eta}\left[2\langle\xi, \eta\rangle+\left\langle\left(\sigma_{1}^{-1}-\sigma_{2}^{-1}\right)^{-1} \eta, \eta\right\rangle-\theta_{1} h_{\mathrm{c}}(\eta)\right],
$$

where $h_{\mathrm{c}}(\eta)$ is defined by

$$
h_{\mathrm{c}}(\eta)=\inf _{|k|=1}\left|\pi_{\sigma_{1}^{-1 / 2} W(k)} \sigma_{1}^{\frac{1}{2}} \eta\right|^{2} .
$$

The proof is parallel to that of $(6.1)$. Optimality is proved using the complementary energy version of the layering formula (6.11), with the roles of $\sigma_{1}$ and $\sigma_{2}$ reversed.

It was shown in [29] that the Hashin-Shtrikman upper bounds on $\sigma^{*}$ are equivalent to the lower bounds on $\left(\sigma^{*}\right)^{-1}$ and the lower bounds on $\sigma^{*}$ are equivalent to the upper bounds on $\left(\sigma^{*}\right)^{-1}$. Thus, (2.3) and (6.16) are equivalent, as are (6.1) and (6.9). Let us make the connection between (6.1) and (6.9) explicit, by what amounts to a specialization of the argument in [29]. (An alternative, more general but more abstract, link is offered by Theorem 8.2.)

Notice that (6.1) makes use of $h(\eta)$, while (6.9) makes use of $g_{\mathrm{c}}(\eta)$. The key to linking the bounds, of course, is to relate these two functions. For any $k \neq 0$ and any $\eta$ we have

$$
\left|\pi_{\sigma_{2}^{-1 / 2} W(k)} \sigma_{2}^{\frac{1}{2}} \eta\right|^{2}+\left|\pi_{\sigma_{2}^{1 / 2} V(k)} \sigma_{2}^{\frac{1}{2}} \eta\right|^{2}=\left|\sigma_{2}^{\frac{1}{2}} \eta\right|^{2},
$$

by (6.8). It follows easily that

$$
g_{\mathrm{c}}(\eta)+h\left(\sigma_{2} \eta\right)=\left\langle\sigma_{2} \eta, \eta\right\rangle .
$$

Next, observe that $(6.1)$ is equivalent to

$$
\theta_{1}\left\langle\left(\sigma_{2}-\sigma^{*}\right)^{-1} \eta, \eta\right\rangle \leq\left\langle\left(\sigma_{2}-\sigma_{1}\right)^{-1} \eta, \eta\right\rangle-\theta_{2} h(\eta)
$$

and (6.9) is equivalent to

$$
\theta_{1}\left\langle\left(\left(\sigma^{*}\right)^{-1}-\sigma_{2}^{-1}\right)^{-1} \eta, \eta\right\rangle \leq\left\langle\left(\sigma_{1}^{-1}-\sigma_{2}^{-1}\right)^{-1} \eta, \eta\right\rangle+\theta_{2} g_{c}(\eta) .
$$


The correspondence between (6.1) and (6.19) is like that between (2.3) and (4.19): the Hashin-Shtrikman bound gives an inequality for every pair of tensors $\xi, \eta$. Optimization over $\eta$ gives (6.1), and optimization over $\xi$ gives (6.19). The correspondence between (6.9) and (6.20) is similar.

It remains to connect (6.19) and (6.20). Replacing $\eta$ by $\sigma_{2}^{-1} \eta$ in (6.20) gives

$$
\begin{aligned}
& \theta_{1}\left\langle\sigma_{2}^{-1}\left(\left(\sigma^{*}\right)^{-1}-\sigma_{2}^{-1}\right)^{-1} \sigma_{2}^{-1} \eta, \eta\right\rangle \\
& \quad \leq\left\langle\sigma_{2}^{-1}\left(\sigma_{1}^{-1}-\sigma_{2}^{-1}\right)^{-1} \sigma_{2}^{-1} \eta, \eta\right\rangle+\theta_{2}\left\langle\sigma_{2}^{-1} \eta, \eta\right\rangle-\theta_{2} h(\eta) .
\end{aligned}
$$

After a bit of algebra, making use of the fact that

$$
\left(\sigma_{1}^{-1}-\sigma_{2}^{-1}\right)^{-1}=\sigma_{2}\left(\sigma_{2}-\sigma_{1}\right)^{-1} \sigma_{1}
$$

and a similar relation with $\sigma_{1}$ replaced by $\sigma^{*}$, one verifies that $(6.21)$ is the same as (6.19).

7. Explicit calculation of $g(\eta)$ and $h(\eta)$ for isotropic reference materials. Our optimal bounds on $\left\langle\sigma^{*} \xi, \xi\right\rangle$ depend on the functions $g$ and $h$, defined by

$$
\begin{aligned}
& g(\eta)=\sup _{|k|=1}\left|\pi_{\sigma_{1}^{1 / 2} V(k)} \sigma_{1}^{-\frac{1}{2}} \eta\right|^{2}, \\
& h(\eta)=\inf _{|k|=1}\left|\pi_{\sigma_{2}^{1 / 2} V(k)} \sigma_{2}^{-\frac{1}{2}} \eta\right|^{2} .
\end{aligned}
$$

If the bounds are to be evaluated either analytically or numerically, it is obviously important to understand these functions.

Actually, $g(\eta)$ has been studied quite extensively in the metallurgical literature; see [23] and the references given there. The goal of that work is to explain certain microstructures which arise in coherent mixtures of distinct elastic phases. According to the theory of Khachaturyan and Roitburd [18, 34], if two phases have different stress-free strains but identical elastic moduli then elastic energy is minimized by a microstructure of plate-like inclusions. The orientation of the inclusions is determined by (7.1); they should be normal to a direction $k$ which is extremal for (7.1). A mathematical explanation of this theory is presented in [20].

The goal of this section is to give explicit formulas for $g(\eta)$ and $h(\eta)$ when $\sigma_{1}$ and $\sigma_{2}$ are isotropic. These formulas will be used in [1] to evaluate some of the optimal bounds explicitly.

Our upper and lower bounds on complementary energy make use of two additional functions $g_{\mathrm{c}}(\eta)$ and $h_{\mathrm{c}}(\eta)$, defined by (6.10) and (6.17). They are determined by $g$ and $h$, however: (6.18) gives $g_{c}$ in terms of $h$, and the analogous relation

$$
h_{\mathrm{c}}(\eta)+g\left(\sigma_{1} \eta\right)=\left\langle\sigma_{1} \eta, \eta\right\rangle
$$

gives $h_{\mathrm{c}}$ in terms of $g$. Therefore, it is not necessary to devote separate attention to $g_{\mathrm{c}}$ or $h_{\mathrm{c}}$.

For the remainder of this section, we let $\sigma$ denote the isotropic Hooke's law

$$
\sigma \xi=\kappa(\operatorname{tr} \xi) I+2 \mu\left(\xi-\frac{1}{N}(\operatorname{tr} \xi) I\right)
$$


with bulk modulus $\kappa$ and shear modulus $\mu$. This law can also be expressed as

$$
\sigma \xi=2 \mu \xi+\lambda(\operatorname{tr} \xi) I
$$

with $\lambda=\kappa-2 \mu / N$. We require $\kappa>0, \mu>0$ so that $\sigma$ determines a positive definite quadratic form on second-order tensors. Most elastic materials also satisfy $\lambda>0$ (this is equivalent to Poisson's ratio being positive); however, we do not assume $\lambda>0$, except as explicitly stated below.

LeMMA 7.1. For any symmetric tensor $\eta$ and any unit vector $k$,

$$
\left|\pi_{\sigma^{\frac{1}{2}} V(k)} \sigma^{-\frac{1}{2}} \eta\right|^{2}=\frac{1}{\mu}\left[|\eta k|^{2}-\langle\eta k, k\rangle^{2}\right]+\frac{1}{\lambda+2 \mu}\langle\eta k, k\rangle^{2} .
$$

Proof. This is classical; it amounts to calculating the Fourier transform of the Green's function for linear elasticity. See, for example, Lemma 4.2 of [20] for a complete proof.

To calculate $g(\eta)$ or $h(\eta)$, we must maximize or minimize the right-hand side of (7.6) over all unit vectors $k$. We do $h$ first, since it is easier.

Proposition 7.2. Let $\sigma$ be isotropic, and let $\eta$ have eigenvalues $\eta_{1}, \ldots, \eta_{N}$. Then

$$
\inf _{|k|=1}\left|\pi_{\sigma^{1 / 2} V(k)} \sigma^{-\frac{1}{2}} \eta\right|^{2}=\frac{1}{\lambda+2 \mu} \min _{1 \leq i \leq N}\left\{\eta_{i}^{2}\right\} .
$$

Proof. Our goal is to minimize

$$
G(k)=\frac{1}{\mu}\left[|\eta k|^{2}-\langle\eta k, k\rangle^{2}\right]+\frac{1}{\lambda+2 \mu}\langle\eta k, k\rangle^{2}
$$

with $\eta$ held fixed, subject to $|k|=1$. By the method of Lagrange multipliers, at any critical point $k$ we have

$$
\frac{1}{\mu}\left[\eta^{2} k-2\langle\eta k, k\rangle \eta k\right]+\frac{2}{\lambda+2 \mu}\langle\eta k, k\rangle \eta k=c k
$$

for some $c \in \mathbf{R}$. Since $\eta^{2} k$ is a linear combination of $k$ and $\eta k$, the subspace spanned by $\{k, \eta k\}$ is invariant under multiplication by $\eta$. Thus $\eta$ is diagonalizable on this subspace, and we may write $k=\alpha_{i} \nu_{i}+\alpha_{j} \nu_{j}$, where $\nu_{i}$ and $\nu_{j}$ are eigenvectors of $\eta$ associated to eigenvalues $\eta_{i}$ and $\eta_{j}$, and $\alpha_{i}^{2}+\alpha_{j}^{2}=1$. The relation (7.9) becomes

$$
\frac{1}{\mu}\left[\eta_{p}^{2} \alpha_{p}-2\left(\eta_{i} \alpha_{i}^{2}+\eta_{j} \alpha_{j}^{2}\right) \eta_{p} \alpha_{p}\right]+\frac{2}{\lambda+2 \mu}\left(\eta_{i} \alpha_{i}^{2}+\eta_{j} \alpha_{j}^{2}\right) \eta_{p} \alpha_{p}=c \alpha_{p}
$$

for $p=i, j$.

If $\alpha_{i}=0$ then $k$ is an eigenvector of $\eta$ associated to eigenvalue $\eta_{j}$, and

$$
G(k)=\frac{\eta_{j}^{2}}{\lambda+2 \mu}
$$

Similarly, if $\alpha_{j}=0$, we get $G(k)=\eta_{i}^{2} /(\lambda+2 \mu)$. If $\eta_{i}=\eta_{j}$ then $k$ is once again an eigenvector of $\eta$ and (7.11) holds. 
Suppose now that $\alpha_{i} \neq 0, \alpha_{j} \neq 0$, and $\eta_{i} \neq \eta_{j}$. Then, subtracting one component of (7.10) from the other gives

$$
\eta_{i} \alpha_{i}^{2}+\eta_{j} \alpha_{j}^{2}=\frac{\lambda+2 \mu}{2(\lambda+\mu)}\left(\eta_{i}+\eta_{j}\right)
$$

Bearing in mind that $\alpha_{i}^{2}+\alpha_{j}^{2}=1,(7.12)$ yields

$$
\alpha_{i}^{2}=\frac{(\lambda+2 \mu) \eta_{i}-\lambda \eta_{j}}{2(\lambda+\mu)\left(\eta_{i}-\eta_{j}\right)}, \quad \alpha_{j}^{2}=\frac{(\lambda+2 \mu) \eta_{j}-\lambda \eta_{i}}{2(\lambda+\mu)\left(\eta_{j}-\eta_{i}\right)} .
$$

This gives, after some calculation,

$$
G(k)=\frac{1}{4 \mu}\left(\eta_{i}-\eta_{j}\right)^{2}+\frac{1}{4(\mu+\lambda)}\left(\eta_{i}+\eta_{j}\right)^{2} .
$$

One verifies easily that (7.14) is larger than both $\eta_{i}^{2} /(\lambda+2 \mu)$ and $\eta_{j}^{2} /(\lambda+2 \mu)$, so the minimum is never at a point of this type.

In conclusion, the minimum is achieved when $k$ is an eigenvector of $\eta$ associated with an eigenvalue of least absolute value. The value is given by (7.11), and this yields (7.7).

Now we turn to $g(\eta)$. Of course, most of the work has already been done, since we have identified all the critical points of $G(k)$.

Proposition 7.3. Let $\sigma$ be isotropic, and let $\eta$ have eigenvalues $\eta_{1}, \ldots, \eta_{N}$. Then

$$
\sup _{|k|=1}\left|\pi_{\sigma^{1 / 2} V(k)} \sigma^{-\frac{1}{2}} \eta\right|^{2}=\operatorname{Max}\left\{\frac{\max _{i} \eta_{i}^{2}}{\lambda+2 \mu}, \max _{C_{i j}}\left[\frac{\left(\eta_{i}-\eta_{j}\right)^{2}}{4 \mu}+\frac{\left(\eta_{i}+\eta_{j}\right)^{2}}{4(\lambda+\mu)}\right]\right\},
$$

where $C_{i j}$ is the condition

$$
\eta_{j} \geq \frac{\lambda+2 \mu}{2(\lambda+\mu)}\left(\eta_{i}+\eta_{j}\right) \geq \eta_{i}
$$

Proof. Our goal is to maximize the function $G(k)$, defined by (7.8), over all unit vectors $k$. The proof of Proposition 7.2 identified all the critical points of $G$. They fall into two classes:

(i) eigenvectors of $\eta$,

(ii) linear combinations of two eigenvectors $\nu_{i}$ and $\nu_{j}$ of the form $k=\alpha_{i} \nu_{i}+$ $\alpha_{j} \nu_{j}$, with $\alpha_{i}$ and $\alpha_{j}$ satisfying (7.13).

The first class carries no implicit restriction: every eigenvector is a critical point. The second class, however, is restricted by the observation that $\alpha_{i}^{2}$ and $\alpha_{j}^{2}$ must be nonnegative. Thus, from (7.13), only pairs satisfying

$$
\frac{(\lambda+2 \mu) \eta_{i}-\lambda \eta_{j}}{\eta_{i}-\eta_{j}} \geq 0, \quad \frac{(\lambda+2 \mu) \eta_{j}-\lambda \eta_{i}}{\eta_{j}-\eta_{i}} \geq 0
$$

can arise. We may assume without loss of generality that $\eta_{i}<\eta_{j}$, and then (7.17) is equivalent to (7.16). Since the maximum is achieved at some critical point, this leads to (7.15).

Proposition 7.3 reduces the calculation of $g(\eta)$ from a continuous maximization (over the sphere $|k|=1$ ) to a discrete one. If the spatial dimension is $N=2$ or if Lamé's modulus $\lambda$ is nonnegative, then we can simplify the formula further. 
Proposition 7.4. Assume that either $N=2$ or else $\lambda \geq 0$. Label the eigenvalues of $\eta$ so that

$$
\eta_{1} \leq \cdots \leq \eta_{N}
$$

Then the right-hand side of (7.15) equals

$$
\begin{cases}\frac{\left(\eta_{1}-\eta_{N}\right)^{2}}{4 \mu}+\frac{\left(\eta_{1}+\eta_{N}\right)^{2}}{4(\lambda+\mu)} & \text { if } \eta_{N} \geq \frac{\lambda+2 \mu}{2(\lambda+\mu)}\left(\eta_{1}+\eta_{N}\right) \geq \eta_{1}, \\ \frac{\eta_{1}^{2}}{\lambda+2 \mu} & \text { if } \eta_{1}>\frac{\lambda+2 \mu}{2(\lambda+\mu)}\left(\eta_{1}+\eta_{N}\right), \\ \frac{\eta_{N}^{2}}{\lambda+2 \mu} & \text { if } \eta_{N}<\frac{\lambda+2 \mu}{2(\lambda+\mu)}\left(\eta_{1}+\eta_{N}\right) .\end{cases}
$$

Proof. Suppose first that $N=2$. We observed in proving Proposition 7.2 that the value of $G$ at any type (ii) critical point is greater than the value at every type (i) critical point. Hence the maximum is certain to be

$$
\frac{1}{4 \mu}\left(\eta_{1}-\eta_{2}\right)^{2}+\frac{1}{4(\lambda+\mu)}\left(\eta_{1}+\eta_{2}\right)^{2}
$$

if condition $C_{12}$ applies, i.e., if $\eta_{2} \geq[(\lambda+2 \mu) / 2(\lambda+\mu)]\left(\eta_{1}+\eta_{2}\right) \geq \eta_{1}$. This gives the first regime of (7.19). If condition $C_{12}$ fails then either

$$
\eta_{1}>\frac{\lambda+2 \mu}{2(\lambda+\mu)}\left(\eta_{1}+\eta_{2}\right)
$$

or

$$
\eta_{2}<\frac{\lambda+2 \mu}{2(\lambda+\mu)}\left(\eta_{1}+\eta_{2}\right)
$$

We claim that (7.20) implies $\eta_{1}^{2}>\eta_{2}^{2}$ and (7.21) implies $\eta_{2}^{2}>\eta_{1}^{2}$. Indeed (7.20) yields

$$
\eta_{1} \leq \eta_{2}<\frac{\lambda}{\lambda+2 \mu} \eta_{1}
$$

(we use here that $\lambda+\mu=\kappa>0$ when $N=2$, regardless of the sign of $\lambda$ ). If $\lambda \geq 0$ then (7.22) forces $\eta_{1} \leq \eta_{2} \leq 0$, whence $\eta_{1}^{2} \geq \eta_{2}^{2}$. If $\lambda \leq 0$ then (7.22) is consistent with either $\eta_{1} \leq \eta_{2} \leq 0$ or $\eta_{1} \leq 0 \leq \eta_{2}$. But in the latter case we have $\eta_{2} \leq\left|\eta_{1}\right|$, since $\lambda /(\lambda+2 \mu)>-1$. Thus (7.20) implies $\eta_{1}^{2}>\eta_{2}^{2}$, regardless of the sign of $\lambda$. This gives the second regime of (7.19). The proof that (7.21) implies $\eta_{2}^{2}>\eta_{1}^{2}$ is similar, and it gives the third regime of (7.19).

Now we suppose that $N>2$, but we assume $\lambda>0$. Two eigenvalues $\eta_{j} \geq \eta_{i}$ satisfy the condition $C_{i j}$ exactly if

$$
(\lambda+2 \mu) \eta_{i} \leq \lambda \eta_{j}, \quad \lambda \eta_{i} \leq(\lambda+2 \mu) \eta_{j} .
$$

Since $\lambda \geq 0$, it is easy to see that if $\left(\eta_{j}, \eta_{i}\right)$ satisfy (7.23) then so do $\left(\eta_{N}, \eta_{i}\right)$, $\left(\eta_{j}, \eta_{1}\right)$, and $\left(\eta_{N}, \eta_{1}\right)$. Moreover, (7.23) and the relation $\eta_{N} \geq \eta_{j}$ yield

$$
\frac{1}{4 \mu}\left(\eta_{i}-\eta_{j}\right)^{2}+\frac{1}{4(\mu+\lambda)}\left(\eta_{i}+\eta_{j}\right)^{2} \leq \frac{1}{4 \mu}\left(\eta_{i}-\eta_{N}\right)^{2}+\frac{1}{4(\mu+\lambda)}\left(\eta_{i}+\eta_{N}\right)^{2} .
$$


Similarly, (7.23) and the relation $\eta_{i} \geq \eta_{1}$ yield

$$
\frac{1}{4 \mu}\left(\eta_{i}-\eta_{N}\right)^{2}+\frac{1}{4(\mu+\lambda)}\left(\eta_{i}+\eta_{N}\right)^{2} \leq \frac{1}{4 \mu}\left(\eta_{1}-\eta_{N}\right)^{2}+\frac{1}{4(\mu+\lambda)}\left(\eta_{1}+\eta_{N}\right)^{2} .
$$

Thus the right-hand side of (7.15) equals

$$
\begin{cases}\frac{1}{4 \mu}\left(\eta_{1}-\eta_{N}\right)^{2}+\frac{1}{4(\lambda+\mu)}\left(\eta_{1}+\eta_{N}\right)^{2} & \text { if } C_{1 N} \text { holds, } \\ \frac{1}{\lambda+2 \mu} \max _{i} \eta_{i}^{2} & \text { if } C_{1 N} \text { fails. }\end{cases}
$$

The case when $C_{1 N}$ holds gives the first regime of (7.19). If $C_{1 N}$ fails then either

$$
\eta_{1}>\frac{\lambda+2 \mu}{2(\lambda+\mu)}\left(\eta_{1}+\eta_{N}\right)
$$

or

$$
\eta_{N}<\frac{\lambda+2 \mu}{2(\lambda+\mu)}\left(\eta_{1}+\eta_{N}\right)
$$

The argument presented above for $N=2$ shows (in any dimension, if $\lambda \geq 0$ ) that (7.25) implies $\eta_{1}^{2}>\eta_{N}^{2}$ and (7.26) implies $\eta_{N}^{2}>\eta_{1}^{2}$. These yield the remaining regimes of (7.19).

8. Some general properties of optimal bounds. This section establishes two general properties of optimal bounds. Well-ordering is not assumed, and the arguments do not depend on having some type of "formula" for the bounds. The results are presented for simplicity in the setting of two-component composites; however, the proofs generalize easily to other situations.

Our first result asserts the continuity of an optimal bound with respect to all its parameters. As a typical application, recall from Secs. 2 and 3 that it is technically simpler to consider two "strictly well-ordered" materials $\sigma_{1}<\sigma_{2}$ rather than two "weakly well-ordered ones" $\sigma_{1} \leq \sigma_{2}$. By Proposition 8.1, it is actually sufficient to prove the bound for strictly well-ordered materials: the weakly well ordered case then follows by continuity.

Proposition 8.1. Let $\sigma_{1}$ and $\sigma_{2}$ be two Hooke's laws, not necessarily well ordered. Suppose that

$$
\left\langle\sigma^{*} \xi, \xi\right\rangle \geq f_{-}\left(\sigma_{1}, \sigma_{2}, \theta_{1}, \theta_{2}, \xi\right)
$$

is the optimal lower bound on $\left\langle\sigma^{*} \xi, \xi\right\rangle$, for composites $\sigma^{*}$ made from $\sigma_{1}$ and $\sigma_{2}$ in volume fractions $\theta_{1}$ and $\theta_{2}$ respectively. Then $f_{-}$is a continuous function of all its variables. A similar assertion holds for upper bounds, complementary energy bounds, and bounds on sums of energies or complementary energies.

Proof. First let us prove continuity with respect to $\xi$. Given $\xi^{\prime}$ near $\xi$ and any $\varepsilon>0$, consider a microstructure $\sigma(y)$ which almost achieves the bound:

$$
\left\langle\sigma^{*} \xi, \xi\right\rangle \leq f_{-}\left(\sigma_{1}, \sigma_{2}, \theta_{2}, \xi\right)+\varepsilon
$$

For any fixed microstructure we have

$$
\left\langle\sigma^{*} \xi^{\prime}, \xi^{\prime}\right\rangle \leq\left\langle\sigma^{*} \xi, \xi\right\rangle+C\left|\xi^{\prime}-\xi\right|
$$


where $C$ depends only on $|\xi|$ and on a pointwise upper bound for $\sigma(y)$. So the microstructure that achieves (8.2) also satisfies

$$
\left\langle\sigma^{*} \xi^{\prime}, \xi^{\prime}\right\rangle \leq f_{-}\left(\sigma_{1}, \sigma_{2}, \theta_{1}, \theta_{2}, \xi\right)+\varepsilon+C\left|\xi-\xi^{\prime}\right| .
$$

It follows as $\varepsilon \rightarrow 0$ that

$$
f_{-}\left(\sigma_{1}, \sigma_{2}, \theta_{1}, \theta_{2}, \xi^{\prime}\right) \leq f_{-}\left(\sigma_{1}, \sigma_{2}, \theta_{1}, \theta_{2}, \xi\right)+C\left|\xi-\xi^{\prime}\right| .
$$

Reversing the roles of $\xi$ and $\xi^{\prime}$, we conclude that $f_{-}$is locally Lipschitz continuous in $\xi$.

Next we prove continuity with respect to $\sigma_{1}$ and $\sigma_{2}$. For any $\varepsilon>0$, consider a microstructure $\sigma(y)=\sigma_{1} \chi_{1}(y)+\sigma_{2} \chi_{2}(y)$ satisfying (8.2). For any $\sigma_{1}^{\prime}$ and $\sigma_{2}^{\prime}$ close to $\sigma_{1}$ and $\sigma_{2}$, consider the microstructure $\sigma^{\prime}(y)=\sigma_{1}^{\prime} \chi_{1}(y)+\sigma_{2}^{\prime} \chi_{2}(y)$. By an application of Meyers's theorem,

$$
\left|\sigma^{*}-\left(\sigma^{\prime}\right)^{*}\right| \leq C\left(\left|\sigma_{1}-\sigma_{1}^{\prime}\right|+\left|\sigma_{2}-\sigma_{2}^{\prime}\right|\right)^{\alpha}
$$

for some constants $C>0, \alpha>0$. (Meyers's theorem gives an $L^{p}$ bound for the strain associated to the solution of any "cell problem", with $p>2$ depending only on pointwise upper and lower ellipticity bounds. A proof for linear elastostatics can be given by arguing as in [12, Chapter 5].) We deduce from (8.2) and (8.3) that

$$
f_{-}\left(\sigma_{1}^{\prime}, \sigma_{2}^{\prime}, \theta_{1}, \theta_{2}, \xi\right) \leq f_{-}\left(\sigma_{1}, \sigma_{2}, \theta_{1}, \theta_{2}, \xi\right)+C\left(\left|\sigma_{1}-\sigma_{1}^{\prime}\right|+\left|\sigma_{2}-\sigma_{2}^{\prime}\right|\right)^{\alpha} .
$$

This gives Hölder continuity in $\sigma_{1}$ and $\sigma_{2}$.

Continuity in $\theta_{1}$ is similar. (Note that $\theta_{2}=1-\theta$ is not an independent variable.) Given $\sigma(y)=\sigma_{1} \chi_{1}(y)+\sigma_{2} \chi_{2}(y)$ satisfying (8.2), a comparison microstructure $\sigma^{\prime}(y)=\sigma_{1} \chi_{1}^{\prime}(y)+\sigma_{2} \chi_{2}^{\prime}(y)$ can be chosen so that

$$
\chi_{1}^{\prime}(y)=\chi_{1}(y) \text { except on volume fraction }\left|\theta_{1}^{\prime}-\theta_{1}\right| \text {. }
$$

Meyers's theorem yields

$$
\left|\sigma^{*}-\left(\sigma^{\prime}\right)^{*}\right| \leq C\left|\theta_{1}-\theta_{1}^{\prime}\right|^{\alpha},
$$

leading to the Hölder continuity of $f_{-}$with respect to $\theta_{1}$.

The other bounds (upper bounds, complementary energy, etc.) can obviously be treated in exactly the same way.

Our second result links the two upper bounds (on energy and complementary energy) and the corresponding lower bounds, in pairs. We already drew such a connection in Sec. 6, using the explicit formulas established in the well-ordered case. The following argument has the advantage of applying in general, even if $\sigma_{1}$ and $\sigma_{2}$ are not well-ordered, and even if the optimal bounds are not known explicitly.

Proposition 8.2. Consider composites made from any two elastic materials $\sigma_{1}$ and $\sigma_{2}$ in volume fractions $\theta_{1}$ and $\theta_{2}$. Let

$$
f_{-}(\xi) \leq\left\langle\sigma^{*} \xi, \xi\right\rangle \leq f_{+}(\xi)
$$

and

$$
f_{-}^{\mathrm{c}}(\xi) \leq\left\langle\left(\sigma^{*}\right)^{-1} \xi, \xi\right\rangle \leq f_{+}^{\mathrm{c}}(\xi)
$$


be the optimal bounds on elastic energy and complementary energy. Then $\frac{1}{2} f_{+}^{\mathrm{c}}$ and $\frac{1}{2} f_{-}$are the Fenchel transforms of one another, as are $\frac{1}{2} f_{-}^{\mathrm{c}}$ and $\frac{1}{2} f_{+}$. In particular, all four functions are convex with respect to $\xi$.

Proof. Our starting point is the optimal lower bound on elastic energy

$$
\left\langle\sigma^{*} \xi, \xi\right\rangle \geq f_{-}(\xi) \text {. }
$$

We shall show that

$$
\left\langle\left(\sigma^{*}\right)^{-1} \zeta, \zeta\right\rangle \leq \sup _{\xi}\left[2\langle\xi, \zeta\rangle-f_{-}(\xi)\right]
$$

is the optimal upper bound on complementary energy. (It should be emphasized that $f_{-}$is not assumed to be convex.)

As a first step, let us show that (8.7) is a valid upper bound. We have

$$
\left\langle\left(\sigma^{*}\right)^{-1} \zeta, \zeta\right\rangle=\sup _{\xi}\left[2\langle\xi, \eta\rangle-\left\langle\sigma^{*} \xi, \xi\right\rangle\right]
$$

since $\sigma^{*}$ is a positive quadratic form. Relation (8.7) follows immediately from (8.6) and $(8.8)$.

Now let us show that (8.7) is optimal. We know from Proposition 8.1 that $f_{-}$is a continuous function of $\xi$. Basic facts of homogenization show that it satisfies

$$
\begin{gathered}
c_{1}|\xi|^{2} \leq f_{-}(\xi) \leq c_{2}|\xi|^{2}, \\
f_{-}(t \xi)=t^{2} f_{-}(\xi) .
\end{gathered}
$$

Therefore, the supremum in (8.7) is achieved, say at $\xi^{*}$. (We do not assert that $\xi^{*}$ is unique, since $f_{-}$is not known at this stage to be convex.) Taking the first variation in direction $\xi^{*}$ gives

$$
\left.\frac{d}{d t}\right|_{t=1}\left[2\left\langle t \xi^{*}, \zeta\right\rangle-t^{2} f_{-}\left(\xi^{*}\right)\right]=0,
$$

whence $\left\langle\xi^{*}, \zeta\right\rangle=f_{-}\left(\xi^{*}\right)$. Thus (8.7) actually asserts that

$$
\left\langle\left(\sigma^{*}\right)^{-1} \zeta, \zeta\right\rangle \leq\left\langle\xi^{*}, \zeta\right\rangle
$$

Consider a microstructure that approaches equality in (8.6) with $\xi=\xi^{*}$, i.e., such that

$$
\left\langle\sigma^{*} \xi^{*}, \xi^{*}\right\rangle \leq f_{-}\left(\xi^{*}\right)+\varepsilon .
$$

Its effective tensor satisfies

$$
\begin{aligned}
\left\langle\left(\sigma^{*}\right)^{-1} \zeta, \zeta\right\rangle & \geq 2\left\langle\xi^{*}, \zeta\right\rangle-\left\langle\sigma^{*} \xi^{*}, \xi^{*}\right\rangle \\
& \geq 2\left\langle\xi^{*}, \zeta\right\rangle-f_{-}\left(\xi^{*}\right)-\varepsilon \\
& \geq\left\langle\xi^{*}, \zeta\right\rangle-\varepsilon .
\end{aligned}
$$

As $\varepsilon \rightarrow 0$, this shows that $(8.11)$ is the optimal upper bound on $\left\langle\left(\sigma^{*}\right)^{-1} \zeta, \zeta\right\rangle$. In other words,

$$
f_{+}^{\mathrm{c}}(\zeta)=\sup _{\xi}\left[2\langle\xi, \zeta\rangle-f_{-}(\xi)\right]
$$

We have shown that $\frac{1}{2} f_{+}^{\mathrm{c}}$ is the Fenchel transform of $\frac{1}{2} f_{-}(\xi)$. 
Similar arguments show that

$$
\begin{aligned}
f_{-}^{\mathrm{c}}(\zeta) & =\sup _{\xi}\left[2\langle\xi, \zeta\rangle-f_{+}(\xi)\right], \\
f_{-}(\xi) & =\sup _{\zeta}\left[2\langle\zeta, \xi\rangle-f_{+}^{\mathrm{c}}(\zeta)\right], \\
f_{+}(\xi) & =\sup _{\zeta}\left[2\langle\zeta, \xi\rangle-f_{-}^{\mathrm{c}}(\zeta)\right] .
\end{aligned}
$$

Hence all four functions are convex, and they are related in pairs by the Fenchel transform.

\section{REFERENCES}

[1] G. Allaire and R. Kohn, Explicit optimal bounds on the elastic energy of a two-phase composite in two space dimensions, Quart. Appl. Math. LI, 675-699 (1993)

[2] G. Allaire and R. Kohn, Optimal lower bounds on the elastic energy of a composite made from two non-well-ordered isotropic materials, Quart. Appl. Math., in press

[3] G. Allaire and R. Kohn, Optimal design for minimum weight and compliance in plane stress using extremal microstructures, European J. Mech. A Solids, in press

[4] M. Avellaneda, Optimal bounds and microgeometries for elastic two-phase composites, SIAM J. Appl. Math. 47, 1216-1228 (1987)

[5] M. Avellaneda, Bounds on the effective elastic constants of two-phase composite materials, in Nonlinear Partial Differential Equations and Their Applications, College de France Seminar, H. Brezis and J.-L. Lions (Eds.), vol. 10, Longman Scientific, 1991, pp. 1-34

[6] A. Bensoussan, J.-L. Lions, and G. Papanicolaou, Asymptotic Analysis for Periodic Structures, North-Holland, Amsterdam, 1978

[7] F. H. Clarke, Optimization and Non-Smooth Analysis, John Wiley and Sons, New York, 1983

[8] G. Dal Maso and R. Kohn, The local character of G-closure, in preparation

[9] I. Ekeland and R. Temam, Convex Analysis and Variational Problems, North-Holland, Amsterdam, 1976

[10] G. Francfort and J. Marigo, Stable damage evolution in a brittle continuous medium, European J. Mech. A Solids, in press

[11] G. Francfort and F. Murat, Homogenization and optimal bounds in linear elasticity, Arch. Rational Mech. Anal. 94, 307-334 (1986)

[12] M. Giaquinta, Multiple integrals in the Calculus of Variations and Nonlinear Elliptic Systems, Princeton Univ. Press, Princeton, NJ, 1983

[13] L. Gibiansky and A. Cherkaev, Design of composite plates of extremal rigidity, Ioffe Physicotechnical Institute preprint 914,1984; and Microstructures of composites of extremal rigidity and exact estimates of the associated energy density, Ioffe Physicotechnical Institute preprint 1115, 1987 (in Russian)

[14] K. Golden and G. Papanicolaou, Bounds for effective parameters of heterogeneous media by analytic continuation, Comm. Math. Phys. 90, 473-491 (1983)

[15] Z. Hashin and S. Shtrikman, A variational approach to the theory of the elastic behavior of multiphase materials, J. Mech. Phys. Solids 11, 127-140 (1963)

[16] R. Hill, New derivations of some elastic extremum principles, Progress in Applied Mechanics-The Prager Anniversary Volume, Macmillan, New York, 1963, pp. 93-106

[17] Y. Kantor and D. Bergman, Improved rigorous bounds on the effective elastic moduli of a composite material, J. Mech. Phys. Solids 32, 41-62 (1984)

[18] A. Khachaturyan, Theory of Structural Transformations in Solids, John Wiley and Sons, New York, 1983

[19] R. Kohn, Recent progress in the mathematical modeling of composite materials, Composite Material Response: Constitutive Relations and Damage Mechanisms, G. Sih et al. (Eds.), Elsevier, New York, 1988, pp. 155-177

[20] R. Kohn, Relaxation of a double-well energy, Continuum Mechanics and Thermodynamics 3, 193-236 (1991)

[21] R. Kohn and J. Lu, in preparation 
[22] R. Kohn and R. Lipton, Optimal bounds for the effective energy of a mixture of isotropic, incompressible, elastic materials, Arch. Rational Mech. Anal. 102, 331-350 (1988)

[23] E. Kostlan and J. Morris, The preferred habit of a coherent thin-plate inclusion in an anisotropic elastic solid, Acta Metall. 35, 2167-2175 (1987)

[24] R. Lipton, On the behavior of elastic composites with transverse isotropic symmetry, J. Mech. Phys. Solids 39, 663-681 (1991)

[25] R. Lipton, Bounds and perturbation series for incompressible elastic composites with transverse isotropic symmetry, J. Elasticity 27, 193-225 (1992)

[26] K. Lurie and A. Cherkaev, Exact estimates of conductivity of composites formed by two isotropically conducting media taken in prescribed proportion, Proc. Roy. Soc. Edinburgh Sect. A 99, 71-87 (1984)

[27] G. Milton, Modeling the properties of composites by laminates, Homogenization and Effective Moduli of Materials and Media, J. Ericksen et al. (Eds.), Springer-Verlag, New York, 1986, pp. $150-174$

[28] G. Milton, On characterizing the set of possible effective tensors of composites: the variational method and the translation method, Comm. Pure Appl. Math. 43, 63-125 (1990)

[29] G. Milton and R. Kohn, Variational bounds on the effective moduli of anisotropic composites, J. Mech. Phys. Solids 36, 597-629 (1988)

[30] F. Murat, H-convergence, Séminaire d'Analyse Fonctionnelle et Numérique de l'Université d'Alger, mimeographed notes, 1978

[31] F. Murat, $A$ survey on compensated compactness, Contributions to the Modern Calculus of Variations, L. Cesari (Ed.), Pitman, New York, 1987, pp. 145-183

[32] A. Norris, A differential scheme for the effective moduli of composites, Mech. Mater. 4, 1-16 (1985)

[33] G. Papanicolaou and S. Varadhan, Boundary value problems with rapidly oscillating random coefficients, Random Fields, Colloq. Math. Soc. János Bolyai, vol. 27, North-Holland, Amsterdam, 1982, pp. 835-873

[34] A. Roitburd, The domain structure of crystals formed in the solid phase, Sov. Phys.-Solid State 10, 2870-2876 (1969)

[35] E. Sanchez-Palencia, Non homogeneous media and vibration theory, Lecture Notes in Phys., vol. 127, Springer-Verlag, New York, 1980

[36] P. Suquet, Une méthode duale en homogénéisation: Application aux milieux élastiques, J. Mech. Theoret. Appl., special issue, 79-98 (1982)

[37] L. Tartar, Estimation de coefficients homogénéisés, Computing Methods in Applied Sciences and Engineering, R. Glowinski and J.-L. Lions (Eds.), Lecture Notes in Math., vol. 704, SpringerVerlag, New York, 1978, pp. 364-373

[38] L. Tartar, Estimations fines des coefficients homogénéisés, Ennio de Giorgi’s Colloquium, P. Kree (Ed.), Pitman Res. Notes in Math. Ser., vol. 125, Longman Sci. Tech., Harlow, 1985, pp. 168-187

[39] L. Tartar, H-measures and small amplitude homogenization, Random Media and Composites, R. Kohn and G. Milton (Eds.), SIAM, Philadelphia, PA, 1989, pp. 89-99

[40] L. Walpole, On bounds for the overall elastic moduli of inhomogeneous systems. I, J. Mech. Phys. Solids 14, 151-162 (1966)

[41] J. Willis, Variational and related methods for the overall properties of composite materials, C.-S. Yih (Ed.), Adv. in Appl. Mech. 21, 2-78 (1981)

[42] C.-T. Wu and R. McCullough, Constitutive relationships for the heterogeneous materials, Developments in Composite Materials, Applied Science Publishers, 1977, pp. 119-187

[43] V. Zhikov, On estimates for the trace of an averaged tensor, Soviet Math. Dokl. 37, 456-459 (1988)

[44] V. Zhikov, S. Kozlov, O. Oleinik, and K. Ngoan, Averaging and G-convergence of differential operators, Russian Math. Surveys 34, 69-147 (1979) 